bioRxiv preprint doi: https://doi.org/10.1101/802686; this version posted January 18,2020 . The copyright holder for this preprint (which was not certified by peer review) is the author/funder, who has granted bioRxiv a license to display the preprint in perpetuity. It is made available under aCC-BY 4.0 International license.

\title{
Brain aging comprises many modes of structural and functional change with distinct genetic and biophysical associations
}

\author{
Stephen M. Smith ${ }^{a} \quad$ Lloyd T. Elliott $^{b} \quad$ Fidel Alfaro-Almagro $^{a} \quad$ Paul McCarthy $^{a}$ \\ Thomas E. Nichols ${ }^{c, a}$ Gwenaëlle Douaud $^{a}$ Karla L. Miller $^{a}$ \\ ${ }^{a}$ Wellcome Centre for Integrative Neuroimaging (WIN FMRIB), University of Oxford, Oxford, UK \\ ${ }^{b}$ Department of Statistics and Actuarial Science, Simon Fraser University, Vancouver, Canada \\ ${ }^{c}$ Big Data Institute, University of Oxford, Oxford, UK
}

\begin{abstract}
Brain imaging can be used to study how individuals' brains are aging, compared against population norms. This can inform on aspects of brain health; for example, smoking and blood pressure can be seen to accelerate brain aging. Typically, a single "brain age" is estimated per subject, whereas here we we identified 62 modes of subject variability, from 21,407 subjects' multimodal brain imaging data in UK Biobank. The modes represent different aspects of brain aging, showing distinct patterns of functional and structural brain change, and distinct patterns of association with genetics, lifestyle, cognition, physical measures and disease. While conventional brain-age modelling found no genetic associations, 34 modes had genetic associations. We suggest that it is important not to treat brain aging as a single homogeneous process, and that modelling of distinct patterns of structural and functional change will reveal more biologically meaningful markers of brain aging in health and disease.
\end{abstract}

\section{Keywords}

Brain aging, brain imaging, UK Biobank.

Corresponding author: Stephen Smith, steve@fmrib.ox.ac.uk 


\section{Introduction}

Brain imaging can be used to predict "brain age" - the apparent age of individuals' brains - by comparing their imaging data against a normative population dataset. The difference between brain age and actual chronological age (the "delta", or "brain age gap") is often then computed, providing a measure of whether a subject's brain appears to have aged more (or less) than the average age-matched population data. For example, looking at structural magnetic resonance imaging (MRI) data, a high degree of atrophy would cause a subject's brain to appear older than a normal age-matched brain. Estimation of brain age and the delta is of value in studying both normal aging and disease, with some diseases, such as Alzheimer's disease, showing similar patterns of change to that of accelerated healthy aging [Franke et al., 2010 Cole et al., 2017 Cole and Franke, 2017].

The typical approach uses one or more imaging modalities, most commonly using just a single structural image from each subject. The data is then preprocessed, and features identified, for use in the brain age prediction. For example, the structural images may be warped into a standard space, and grey matter segmentation carried out; the voxelwise segmentation values themselves can then be the features. Alternatively, a smaller number of more highly-condensed features may be derived, such as volumes of grey and white matter within multiple brain regions. The resulting dataset, of multiple subjects' feature sets, along with their true ages, is then passed into a supervised-learning algorithm (e.g., regression, support vector machine or deep learning). The algorithm then learns to predict the subjects' ages from their brain imaging features. Finally, the true age is typically subtracted from the estimated brain age, to create a delta, potentially with corrections for biases such as systematic mis-estimation of brain age [Le et al., 2018, Smith et al., 2019].

The imaging feature set can be derived from more than one imaging modality, in which case it can contain information not just about the structural geometric layout of the brain, but also, for example, structural connectivity, white matter microstructure, functional connectivity, iron deposition, and cognitive task activation Groves et al., 2012 Brown et al., 2012 Liem et al., 2017 Vinke et al., 2018 Richard et al., 2018. Such "multimodal" data allows for brain age modelling to take advantage of a richer range of structural and functional measures of change in the brain, but it is still the case that most brain-age modelling only estimates a single overall brain age per individual.

Hence, while the explicit goal of much brain-age research is to obtain a single estimate of brain age (and brain-age delta) per subject, one could nevertheless expect that multiple distinct biological processes contribute to the changes seen in the brain with aging. For example, amounts of physical exercise, intake of alcohol and smoking, dietary patterns, and health factors such as hypertension and obesity, will all likely contribute to the "aging" of the brain, and in potentially different ways. These different factors will likely affect different aspects of the brain's structure and function, as viewed through multiple imaging modalities. Further, different factors affecting brain aging could well have different age dependence - population-averaged aging curves for the different factors could be quite distinct (e.g., with respect to strength and linearity of the age dependence) Groves et al., 2012 Brown et al., 2012, Douaud et al., 2014 Kessler et al., 2016 Vinke et al., 2018 Richard et al., 2018. Different biological factors of brain aging might well also be expected to show distinct genetic influence. The combination of all factors into a single estimate of brain age can be a useful, compact, single summary metric, and is by definition the route by which the most accurate single estimate of a subject's age can be predicted from the imaging data available. However, this may come at the cost of losing important information regarding the distinctions between multiple biological factors occurring, making it harder to understand the (potentially multiple) causes of brain aging.

Here we used six brain imaging modalities from UK Biobank to identify 62 distinct modes of population variation, almost all of which showed significant age effects. In this work we focus on investigating the distinct modes as potentially representing distinct biological factors relating to aging. We aimed to learn about a larger number of distinct modes, and in greater biological depth, than had been previously possible, in part because of the richness of the imaging and non-imaging data available in UK Biobank, and of course due in part to the very large subject numbers. There is nevertheless a link between this approach and the previous literature; one can combine the population modes to produce a single brain-age estimation, which gives similar age prediction accuracy to that derived using standard approaches.

We used the multimodal brain imaging data from 21,407 participants, over the age of 45y, in UK Biobank. Imaging is taking place at 4 sites, with identical imaging hardware, scanner software and protocols (though the subjects used here were from the first two sites). The dataset also includes genetics, lifestyle, cognitive and physical measures, and health outcome information from the healthcare system in the UK. For this work we used 3,913 IDPs (imaging-derived phenotypes, generated by our team on behalf of UK Biobank, and made available to all researchers by UK Biobank). The IDPs are summary measures, each describing a different aspect of brain structure or function. IDPs include functional and structural connectivity between specific pairs of regions, localised tissue microstructure and biological makeup, and the geometry of cortical and subcortical structures.

For our work here, rather than simply feeding all IDPs into one brain age model (e.g., regularised multiple regression), we first 
identify multiple modes that represent different combinations of IDPs that co-vary across subjects. We then use each of these modes separately in simple but standard brain-age modelling. The result is a large number of distinct brain age predictions for each subject, with the goal of each representing a different biological process. We now summarise our approach briefly.

After removal of imaging confound effects (see Methods for details), we used independent component analysis (ICA Hyvärinen, 1999]) to decompose the entire IDP data matrix of $N_{\text {subjects }} \times N_{I D P s}$ into 62 distinct modes of population variation [Kessler et al., 2016, Elliott et al., 2018]. Each mode is described by two vectors. The first is a set of IDP weights, describing which specific aspects of brain structure and function (i.e., which IDPs) are involved in that mode (for example, a given mode might reflect volume of grey matter across various regions involved in language processing). The second is a set of subject weights (one value per subject), describing where in the population distribution a subject lies, in terms of strongly expressing a given mode of variation (e.g., a given subject might have considerably less grey matter in language regions than the population average). These subject-weight vectors (one vector per mode) can be used to help understand the biological meaning of, and causal factors behind, the modes of population variation, by computing associations with non-imaging factors and genetics (a genetic or early-life factor that correlates, across subjects, with our hypothetical mode might suggest biological causes of changes in grey matter volume in the language network). Here we use the subject-weight vectors to study brain aging; virtually all modes show a significant aging effect (Fig. 1), and in this work we study the different aspects of brain aging represented by the 62 modes (as well as 6 clusters of these modes).

Having identified these modes, our modelling of brain age for individual modes follows the same form as commonly used for brain age modelling. We predict subjects' actual age using a given mode's subject-weights-vector, and then subtract the age from the predicted age to obtain the mode-specific brain-age delta. We then use this in our association tests against non-imaging variables and genetics. Hence, instead of using all available data from the brain imaging to obtain a single ("all-in-one") estimate of brain age (and associated delta), we investigate brain aging for each mode separately, to capitalise on the distinct richness of information obtained within separate modes. An indication of the usefulness of doing this can be seen from the fact that many of the modes' delta estimates have significant genetic association (i.e., genetic factors that are significantly driving that aspect of brain aging). By comparison, the all-in-one estimate of brain-age based on a linear combination of modes combines across so many different biological factors that there is no significant, replicated genetic association for the all-in-one delta, despite the overall prediction providing a more accurate estimate of subjects' ages than any one individual mode.

All data is available upon application to UK Biobank. In addition to the main and supplemental figures in this paper, further material is available from the https://www.fmrib.ox.ac.uk/ukbiobank/BrainAgingModes website (see Section 5). This includes: all code written for the work described here; detailed figures, with individual modes' separate genome-wide association study (GWAS) Manhattan plots and resting-state functional MRI (rfMRI) summary brain images; all GWAS summary statistic files; spreadsheets listing all modes' IDP weights, associations with non-imaging-non-genetic variables and peak GWAS associations; and additional genetic analyses including functional annotation, gene expression, associated traits from previous GWAS studies, and genetic heritability/co-heritability results.

\section{Results}

\subsection{Multiple modes and mode-clusters of brain aging}

After discarding outlier data and subjects with high levels of missing/outlier imaging data, we retained data from 18,707 subjects (see Methods). Split-half reproducibility testing $\left(P<10^{-6}\right)$ resulted in estimation of 62 robustly-present ICA modes of population variation. For convenience (and without loss of generality), the modes were inverted where necessary in order for their correlation with age to be positive, and were re-ordered according to decreasing variance explained by a cubic model of age, as reflected in the inset plot of age-mode correlations in Fig. 1 The figure shows the cubic fit of each mode as a function of age (later plots show these fits in more detail and quantitation). The majority of the modes show similar behaviour for females and males, but a few notable exceptions can be seen in supplementary figures (A.3 A.9), as discussed in more detail below.

Using all 62 modes together in an "all-in-one" prediction of overall brain aging, mean absolute delta (the "error" between age and predicted age) was 2.9y. As described in Methods, the all-in-one model is a weighted sum of the 62 modes, where the weights for a given mode are a scalar value that is entirely driven by the unique variance of that mode $\left(\beta_{i}\right.$ for mode $i)$. This unique variance is also referred to as the "partialled" mode, which is calculated by taking a mode's subject weight vector and regressing out the subject vectors of all other modes. Because these partialled modes isolate the unique subject 
bioRxiv preprint doi: https://doi.org/10.1101/802686; this version posted January 18,2020 . The copyright holder for this preprint (which was not certified by peer review) is the author/funder, who has granted bioRxiv a license to display the preprint in perpetuity. It is made available under aCC-BY 4.0 International license.

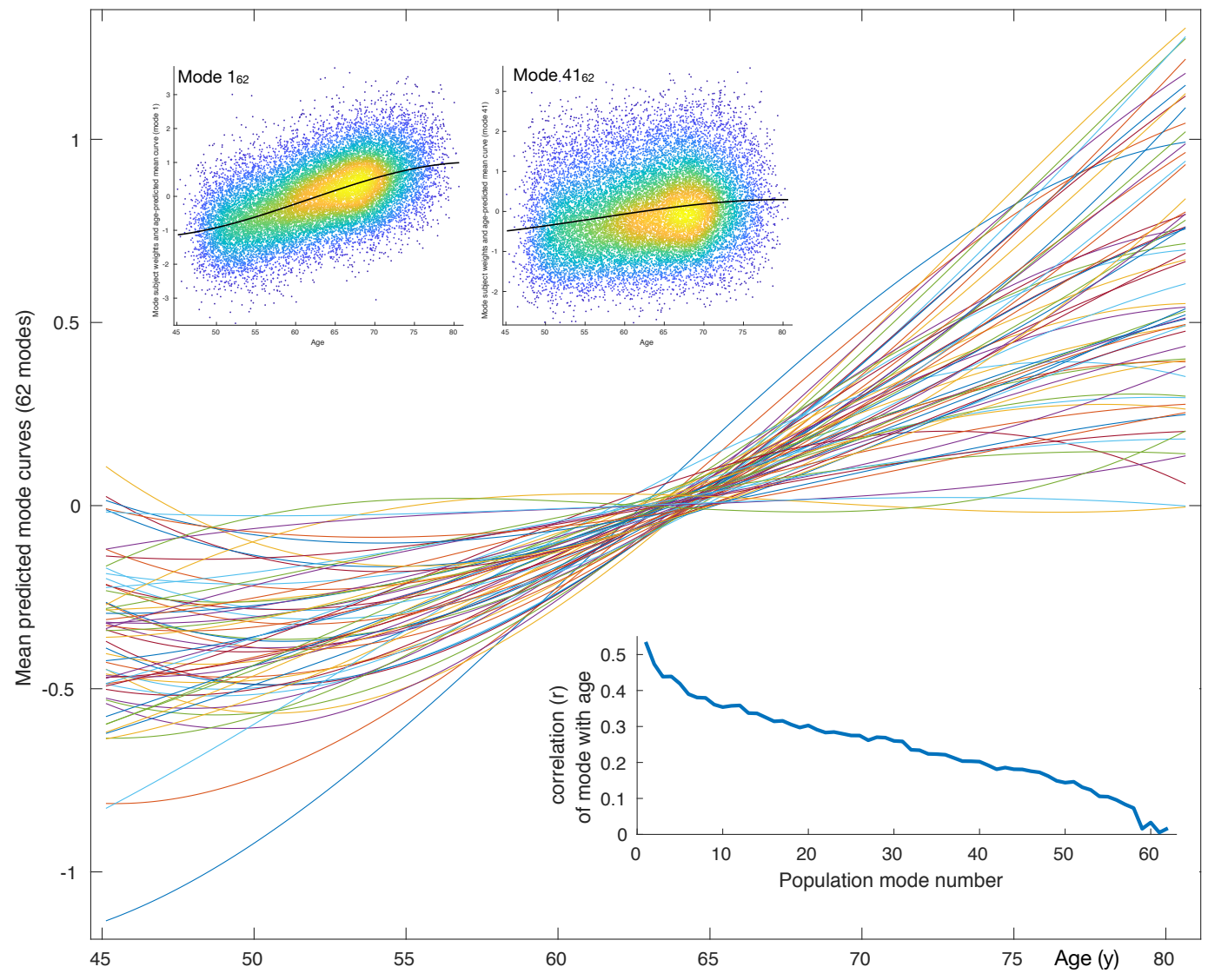

Figure 1: Mean aging curves for the 62 brain-aging modes. The main plot shows the mean aging curves based on a cubic age model - i.e., fitting the subject-weight-vectors from each mode as a function of age, age-square and age-cubed. Therefore the $\mathrm{x}$ axis is age in years, and the $\mathrm{y}$ axis is the unitless values in the original modes' subject-weight-vectors $X_{i}$. The scatter plots show 2 example modes, with their respective mean aging curves shown along with the full data (the modes' subject weights, with a single point for each subject). The inset blue plot shows the strength of age prediction for all modes, quantified simply as correlation of actual age with mode subject-weights.

variance described by a given mode, it is of interest to examine their associations with non-imaging variables, and similarly the associations of partialled deltas. Hence, as seen in Fig. A.2 D, the contribution to age modelling varies highly from mode to mode, driven by the unique variance in each. Several modes have negative $\beta$ weights, meaning that their unique variance is negatively associated with age, even though their original correlation with age was assigned to be positive. Of the 62 modes, 59 correlate significantly with age (at the $P<0.05 / 62$ two-tailed Bonferroni-corrected level), and 29 have a $\beta$ that is significant (i.e., their unique variance has significant age dependence).

In order to help generate more parsimonious descriptions of the 62 modes of brain aging, we investigated whether clustering modes together into a smaller number of mode-clusters could provide a meaningful simplification. Quantitative optimisation of the clustering dimensionality resulted in a meaningful reduction to six mode-clusters (see Methods and Fig. A.1). As with the modes, mode-clusters were defined to correlate positively with age, and sorted in order of decreasing age dependence. As one might expect, there is less redundancy across these 6 mode-clusters (than across the 62 modes), for example, as shown by the fact that the genetic profiles for the partialled 6 mode-cluster deltas are similar to the non-partialled equivalents (Fig. A.12). For clarity, we refer to mode numbers using subscript "62", and to mode-clusters with subscript "6".

\subsection{Mapping of brain-aging modes onto brain structure and function}

Fig. 2 summarises the mapping of modes onto IDPs (different aspects of the brain's structure and function). Each row represents a mode/mode-cluster, and the 3,913 IDPs are arranged into distinct groupings as denoted within the figure. Within 
bioRxiv preprint doi: https://doi.org/10.1101/802686; this version posted January 18,2020 . The copyright holder for this preprint (which was not certified by peer review) is the author/funder, who has granted bioRxiv a license to display the preprint in perpetuity. It is made available under aCC-BY 4.0 International license.

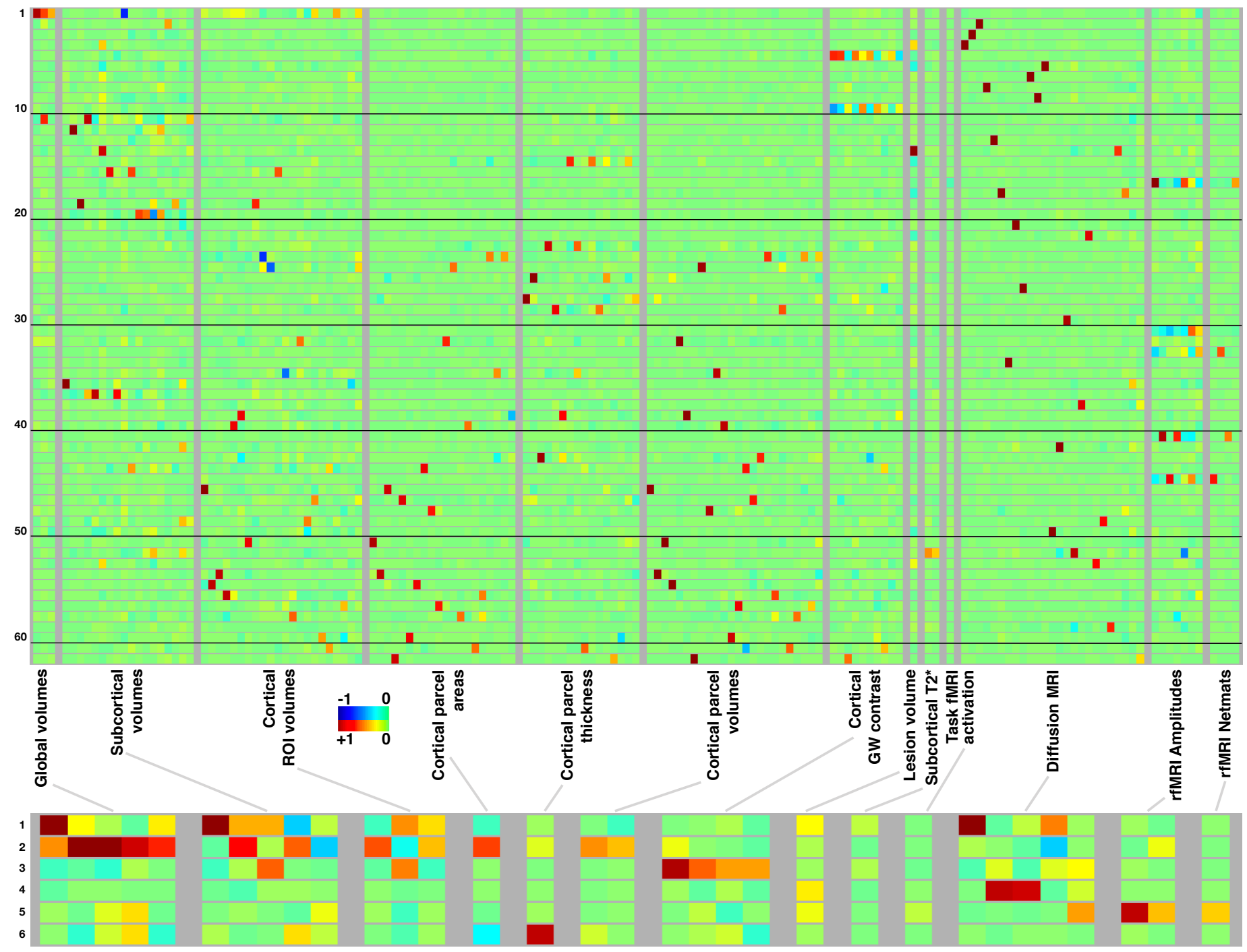

Figure 2: Mapping of 62 brain-aging modes and 6 mode-clusters onto different classes of strucural and functional imaging-derived phenotypes (IDPs). Above: Each row shows the mapping of one brain-aging mode onto the imaging data, with black lines delineating groups of 10 modes for ease of reference. The full plots spanning all 3,913 IDPs are shown in Fig A.10 here, each class of IDPs is reduced using PCA and then ICA to the most representative pseudo-IDPs (see Methods), meaning that each column in the plot relates to the same combination of original IDPs. IDP classes have fewer/greater distinct values here dependent on the number of IDPs in a class, and how highly they correlate with each other. Colour-coded values shown are unitless and mapped into the range $-1: 1$. Below: The equivalent (separately computed) summary figure mapping the 6 mode-clusters onto IDPs.

each grouping, each individual column represents a soft-clustering of highly-correlated IDPs that have similar behaviour to each other (a complete list of the strongest associations between all modes and all IDPs is linked to in Section 5). In most cases individual modes are largely driven by IDPs from a single imaging modality, with a few exceptions such as mode $52_{62}$. Naturally the mode-clusters mix more across modalities. More specific discussion of individual mode and mode-cluster results are given below, in the context of the full set of imaging, non-imaging and genetic associations.

\subsection{Genome-wide associations studies of all brain-aging modes}

We carried out a separate GWAS for the brain-aging delta from each of the 62 modes, and from the 6 mode-clusters. GWAS used 9,812,242 SNPs (single-nucleotide polymorphisms) that passed all quality control tests (see Methods). We also carried 
bioRxiv preprint doi: https://doi.org/10.1101/802686; this version posted January 18,2020 . The copyright holder for this preprint (which was not certified by peer review) is the author/funder, who has granted bioRxiv a license to display the preprint in perpetuity. It is made available under aCC-BY 4.0 International license.

\begin{tabular}{l|rrrr|rr} 
& \multicolumn{5}{|c|}{ Discovery } & \multicolumn{2}{c}{ Validation } \\
Phenotypes & $N_{S N P s}^{7.5}$ & $N_{S N P s}^{9.33}$ & $N_{\text {modes }}^{7.5}$ & $N_{\text {modes }}^{9.33}$ & $N_{S N P s}^{0.05}$ & $N_{\text {modes }}^{0.05}$ \\
\hline 62 modes & 156 & 68 & 50 & 34 & 64 & 34 \\
6 mode-clusters & 33 & 14 & 5 & 3 & 12 & 3 \\
62 modes (partial) & 71 & 29 & 32 & 17 & 27 & 15 \\
6 mode-clusters (partial) & 35 & 12 & 6 & 3 & 11 & 3 \\
all-in-one (62 modes) & 1 & 0 & 1 & 0 & 0 & 0 \\
all-in-one (IDPs) & 3 & 1 & 1 & 1 & 0 & 0
\end{tabular}

Table 1: Summary results of all GWAS of brain-age delta estimates: numbers of supra-threshold SNP clusters from GWAS of all modes (discovery $N=\mathbf{1 0 , 6 1 2}$; validation $N=\mathbf{5 , 3 4 0}$ ). Phenotypes fed into GWAS are grouped and reported on separate rows: the 62 modes' brain-aging deltas, the 6 mode-clusters, the partialled versions of each, and the two separate all-in-one models of brain-age delta that use all 62 modes and all IDPs respectively. The subscripts define whether the counts reported are the number of significant distinct SNP clusters for each phenotype, summed across modes/phenotypes ("SNPs"), or the number of modes/phenotypes with at least one association ("modes"). The superscripts describe the thresholding: either the standard single-GWAS threshold (7.5), the higher Bonferroni-adjusted threshold (9.33), or, in the case of the validation sample, the nominal 0.05 threshold (where here we are just reporting counts of validated associations from the higher discovery threshold).

out GWAS for two "all-in-one" multiple-regression-based estimates of brain-aging delta, one using all 3,913 IDPs in a single prediction of brain aging (with 55-dimensional principal component analysis, PCA, pre-reduction [Smith et al., 2019]), and the other using the 62 modes together (see Methods). The GWAS paradigm we used was similar to that in [Elliott et al., 2018], and associations were tested between these modes and 9,812,242 genetic variants. Results are summarised in Table 1 and Fig. 3. More detailed plots, including separate plots for every mode's GWAS, are provided in Fig. A.12 and Section 5

From the 62 GWAS of modes of brain aging, we found 156 peak associations passing the standard single-GWAS threshold of $-\log _{10} P=7.5$, from the discovery sample of 10,612 subjects (Fig. 3A). Here, "peak associations" means that, in a region of high linkage disequilibrium (LD), we only report the SNP with the highest association with the phenotype, as the associations in the local region are most likely all due to a single genetic effect (see Methods). 68 of these associations passed the more stringent threshold of 9.33 , which increases the standard threshold by a Bonferroni factor of $62+6$ to account for the multiple phenotypes' testing. From the smaller replication dataset of 5,340 subjects, 64 of the 68 peak SNP associations replicated at the $P<0.05$ level. Of the 62 modes, 34 have at least one significant association at the higher threshold, and all of these 34 modes have at least one association in the replication sample.

From the 6 mode-clusters, 14 regions of the genome have significant associations at the higher threshold, 12 of which replicate. Three of the these 6 mode-clusters have at least one significant association, including in replication.

The numbers of associations are lower for the partialled deltas (that reflect unique brain-aging profiles), with the numbers of significant associations approximately halving for the 62 modes, but being reduced only a small amount for the 6 mode-clusters (Table 1).

We also evaluated genetic associations for two "all-in-one" estimations of a single best estimate of brain-age (and associated delta); we used all IDPs in one case, and all modes in the other. This was done with the methods described in Smith et al., 2019]. These two all-in-one brain-age delta estimations showed no genetic assocations that were significant and replicated, consistent with previous GWAS of all-in-one brain-aging modelling [Ning et al., 2018]. This suggests that biological specificity driving the mode/mode-cluster results has been lost (diluted) when generating a single brain-age delta.

Finally, estimates of genetic (SNP) heritability showed that 57 of the 62 modes were significantly heritable, as were all 6 mode-clusters (see Methods and online supplemental results). Estimates of co-heritability with Alzheimer's and Parkinson's disease showed a small number of nominally significant results, but none of these survive multiple comparison correction across modes; this suggests that none of these modes of aging map strongly onto these diseases genetically. 
bioRxiv preprint doi: https://doi.org/10.1101/802686; this version posted January 18,2020 . The copyright holder for this preprint (which was not certified by peer review) is the author/funder, who has granted bioRxiv a license to display the preprint in perpetuity. It is made available under aCC-BY 4.0 International license.

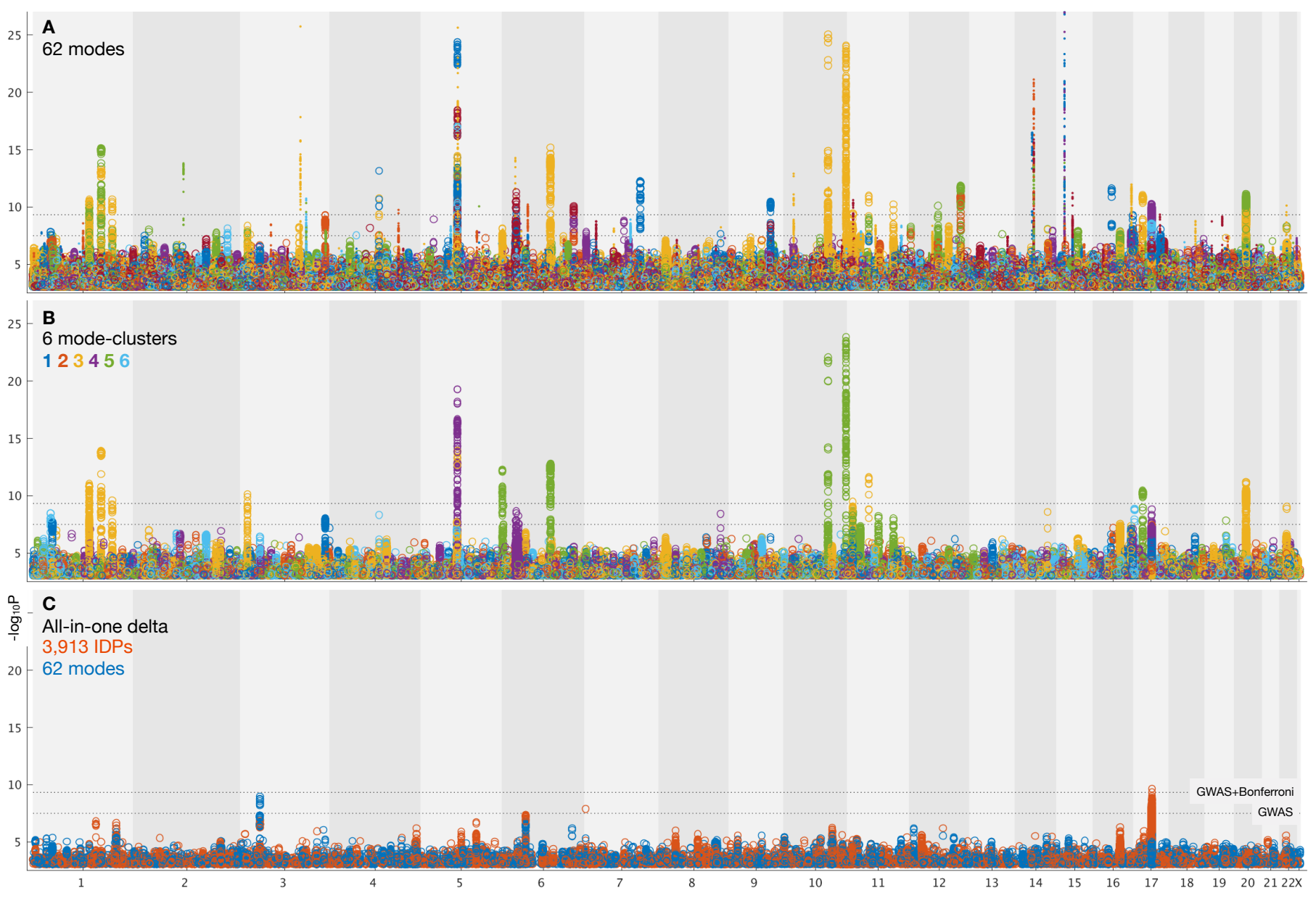

Figure 3: Summary plots for GWAS of brain aging. A) Separate GWAS for each of the 62 modes of brain aging. The y axis is $-\log _{10} P$ (significance of the genetic association) and the $x$ axis is SNPs, arranged according to chromosomes 1:22 and $X$. For convenience of display some points of even higher significance (with redundant content compared with the points seen here) are truncated; for complete plots see Fig. A.12 and for individual plots (one per mode), see Section 5 The lower dotted line shows the standard GWAS threshold correcting for multiple comparisons $\left(-\log _{10} P=7.5\right)$, and the upper line shows the result of an additional Bonferroni correction for the main $62+6$ separate GWAS $\left(-\log _{10} P=9.33\right)$. Circles denote the first 31 brain-aging modes (i.e., those with the strongest aging effect) and dots the next 31 (with weaker aging). B) Separate GWAS for each of the 6 mode-clusters of brain aging. Again, see Fig. A.12 and Section 5 for complete and individual plots. C) GWAS plots for two all-in-one estimates of brain-aging delta (with no points removed). In orange is shown the GWAS for the single delta estimated using all 3,913 IDPs according to the approach in [Smith et al., 2019]. In blue is shown the GWAS for the single delta estimated using the 62 modes. In both cases the richness of genetic associations is clearly greatly reduced, compared with identifying distinct associations for each mode in its own right.

\subsection{Associations of modes with non-imaging variables}

We also computed associations between all modes' deltas and 8,787 nIDPs (non-imaging-derived phenotypes), spanning 16 groups of variable types. These groups include early life factors (e.g., maternal smoking, birth weight), lifestyle factors (e.g., exercise, food, alcohol and tobacco variables), physical body measures (e.g., body size, fat, bone density variables and blood assays), cognitive test scores, and health outcome (including mental health) variables.

Figs. A.13 and A.14 show summarised results, and spreadsheets (Section 5) list every significant association. Below we describe many of these associations in more detail. In general we focus on associations between partialled delta estimates and nIDPs, in order to identify associations specific to the unique brain-age-delta variance in modes. 
bioRxiv preprint doi: https://doi.org/10.1101/802686; this version posted January 18,2020 . The copyright holder for this preprint (which was not certified by peer review) is the author/funder, who has granted bioRxiv a license to display the preprint in perpetuity. It is made available under aCC-BY 4.0 International license.

\subsection{Individual modes: patterns of associations between the aging of the brain's structure and function and life factors, body measures, health outcomes and genetics}

In Table. 2 we list summary results of the strongest patterns of associations with brain-age delta from each mode-cluster and mode. We now expand on some of the more striking patterns in more detail.

Where a SNP discussed below is reported as an expression quantitative trait locus (eQTL) of a gene in the GTEx database [GTEx-Consortium, 2017, this means that variation in this SNP has been found to be highly correlated to variation in the gene expression. Many of the genetic associations described below passed the higher discovery threshold (as well as replicating), but we also discuss some associations that pass the lower (single-phenotype GWAS) threshold if they were also significant in the replication sample.

Mode-cluster $\mathbf{1}_{\mathbf{6}}$, which shows the strongest aging effect of the mode-clusters, is dominated by the volumes of the lateral ventricles and choroid plexus (and its intensity), the microstructure of the fornix and corpus callosum, and the volume of the thalamic nuclei. Notably, and of relevance to further results discussed below, fornix, choroid plexus and corpus callosum are all drained by the superior choroid vein, which runs along the whole length of the choroid plexus, and unites with the superior thalamostriate vein, which itself drains the thalamic nuclei (and striatum). Changes in diffusivity measures in the fornix, a thin tract in the immediate vicinity of the ventricles, may, however, be sensitive, indirect markers of the atrophy of the tract (resulting in a "partial volume" reduction at voxel-level resolution), rather than representing a change to its white matter microstructure.

The non-imaging associations of mode-cluster $1_{6}$ included many modifiable risk factors such as heart rate, smoking, alcohol consumption and diabetes, (as well as taking metformin, a treatment for diabetes, although this is likely an indirect association that is essentially an indicator of the presence of diabetes). It is also associated with various measures related to overall non-fat body size: height, strength, lung capacity, metabolic rate and weight, as well as multiple sclerosis. With regards to cognition, mode-cluster $1_{6}$ was associated with processing speed.

Consistent with the contribution of the identified modifiable risk factors, mode-cluster $1_{6}$ is associated with SNP rs4141741 (MED8), which was significantly correlated in the UK Biobank participants with blood pressure and diagnosed vascular and heart problems. The same SNP is an eQTL in the hippocampus of TIE1, which codes for a protein playing a critical role in angiogenesis and blood vessel stability, and of MED8 in the striatum, both structures being innervated by the superior choroid and thalamostriate veins. Abnormal angiogenesis is also known to contribute to both diabetes and multiple sclerosis, perhaps explaining to some extent our non-imaging association results with both these diseases.

Modes related to mode-cluster $1_{6}$ include $2_{62}$ and $11_{62}$. Mode $11_{62}$ (ventricle volume) is associated with SNP 7:2777917_TA_T (rs1392800372), which is likely in gene GNA12; this has been found to relate to migration of neurons in the developing brain Moers et al., 2008]. This may therefore be relevant in the context of the neural stem cell pool in the subventricular zone [Ruddy et al., 2019], i.e., relating these modes to ventricle size and neuronal development/angiogenesis. In line with mode-cluster $1_{6}$ being dominated by the volumetric measure of CSF (cerebro-spinal fluid, which fills the ventricles), mode $2_{62}$ (fornix MD) is associated with SNP rs150434736 (on chromosome 3, only 17kbp from the 3:190657741_AGT_A/rs147817028 peak in mode-cluster $1_{6}$ ), near gene GMNC; this has been found to be linked to Alzheimer's disease endophenotypes (in particular ptau 181 in CSF) (Cruchaga et al., 2013, Deming et al., 2017.

Mode-cluster $2_{6}$ relates to global measures of grey and white matter volume. It was associated with body-size-related nonimaging measures in common with mode-cluster $1_{6}$, including those of height, weight, strength, metabolic rate and lung function, and also cognitive reaction time.

Several related modes (in particular, $16_{62}, 19_{62}, 24_{62}, 25_{62}, 32_{62}$ and $40_{62}$ ) relate to regional (i.e., more focal) grey matter volume. These modes did not have many nIDP associations (i.e., the nIDP associations for the mode-cluster were largely not regionally specific to individual modes), although mode $32_{62}$ (parietal/occipital volume) was associated with maternal smoking. While mode-cluster $2_{6}$ as a whole did not have strong genetic associations, some of these regional-grey-volume modes did. Modes $16_{62}$ and $19_{62}$ (hippocampus volume) were found to be significantly associated with $H R K$; this is involved in apoptosis/neurogenesis, particularly in adults in hippocampus [Coultas et al., 2007], and expressed (eQTL) in hippocampus. Mode $40_{62}$ (volume of the precuneus cortical region) was associated with DAAM1 [Elliott et al., 2018, Mollink et al., 2019], important for cell polarity and neural development.

While the above modes relate to regionally-specific grey matter volume, there is also involvement (in this mode-cluster) of mode $1_{62}$; this codes for total grey matter volume and is the most strongly age-related mode. This mode is associated with 
bioRxiv preprint doi: https://doi.org/10.1101/802686; this version posted January 18,2020 . The copyright holder for this preprint (which was not certified by peer review) is the author/funder, who has granted bioRxiv a license to display the preprint in perpetuity. It is made available under aCC-BY 4.0 International license.

\begin{tabular}{|c|c|c|c|c|}
\hline $\begin{array}{l}\text { Mode- } \\
\text { cluster }\end{array}$ & Mode-cluster IDPs & Mode-cluster nIDPs & Modes (IDPs and nIDPs) & Genes \\
\hline $1_{6}$ & $\begin{array}{l}\text { CSF/ventricle } \\
\text { volume (both } \\
\text { normalised for head } \\
\text { size and not), fornix } \\
\text { MD. }\end{array}$ & $\begin{array}{l}\text { Non-fat body size (height, fat-free } \\
\text { mass, lung capacity, grip strength, } \\
\text { weight), metabolic rate, head bone } \\
\text { density, cognitive speed, number of } \\
\text { births. Diabetes, alcohol, smoking. }\end{array}$ & $\begin{array}{l}\mathbf{2 6 2}_{62} \text { Fornix MD. } \\
\mathbf{1 1}_{62} \text { CSF/ventricle volume. Head bone area, TV time, cognitive score. } \\
\mathbf{1 2}_{62} \text { Thalamus volume. Body size. } \\
\mathbf{1 3}_{62} \text { Fornix MO. } \\
\mathbf{3 8}_{62} \text { Corona radiata FA. }\end{array}$ & $\begin{array}{l}\text { TIE1 } \\
\text { MED8 } \\
\text { GNA12 } \\
\text { GMNC }\end{array}$ \\
\hline 26 & $\begin{array}{l}\text { Grey volume, white } \\
\text { volume. }\end{array}$ & $\begin{array}{l}\text { Bone density (primarily head), non- } \\
\text { fat body size (height, fat-free mass, } \\
\text { lung capacity, weight), metabolic } \\
\text { rate, ankle width. Number of older } \\
\text { siblings, cognitive speed. }\end{array}$ & $\begin{array}{l}\text { 162 Total grey volume. Bone density (doubled effect in females), age at menopause. } \\
\text { Alcohol, smoking. } \\
\mathbf{1 6}_{62} \text { Amygdala/hippocampus volume. } \mathbf{1 9}_{62} \text { Amygdala/hippocampus volume. } \\
\mathbf{2 4}_{62} \text { Medial-frontal cortical volume. } \mathbf{2 5}_{62} \text { Superior-frontal cortical volume. } \\
\text { 3262 }_{62} \text { Parietal/occipital cortical volume. Maternal smoking, bone density, BMI. } \\
\text { 4062 Precuneus cortical volume. }\end{array}$ & $\begin{array}{l}\text { HRK } \\
\text { DAAM1 } \\
\text { FAM3C } \\
\text { WNT16 } \\
\text { CPED1 }\end{array}$ \\
\hline $3_{6}$ & $\begin{array}{l}\text { T1w cortical grey- } \\
\text { white contrast. }\end{array}$ & BMI, weight, fat mass, haemoglobin. & $\begin{array}{l}\mathbf{5}_{62} \text { Grey-white contrast, frontal. } \\
\mathbf{1 0}_{62} \text { Grey-white contrast, non-frontal. }\end{array}$ & $\begin{array}{ll}\text { CREB3L4 } & \text { CRTC2 } \\
\text { SLC27A3 } & \text { S100A16 } \\
\text { STX6 } & \text { WNTTA } \\
\text { CD82 } & \text { VCAN } \\
\end{array}$ \\
\hline 46 & $\begin{array}{l}\text { MD in all white } \\
\text { matter (particularly } \\
\text { L2, L3). }\end{array}$ & $\begin{array}{l}\text { Blood pressure / hypertension, } \\
\text { multiple sclerosis. }\end{array}$ & $\begin{array}{l}\mathbf{4} 62 \text { SLF MD, white matter lesions. } \\
\mathbf{6}_{62} \text { Superior corona radiata MD. Head bone area, smoking. } \\
\mathbf{7 6 2}_{62} \text { External capsule MD. Blood pressure. } \\
\mathbf{8}_{62} \text { Uncinate MD. } \mathbf{9}_{62} \text { Posterior thalamic radiation / ILF MD. } \\
\mathbf{1 4}_{62} \text { White matter lesions, posterior corona radiata MD. Multiple sclerosis. } \\
\mathbf{2 1}_{62} \text { Cerebral peduncle L2/L3. } \mathbf{2 2} 62 \text { SLFMD. } \mathbf{2 7} 62 \text { Inferior cingulum MD. } \\
\mathbf{3 0}_{62} \text { Superior cingulate gyrus: } L 2 / L 3, M O / F A . \\
\mathbf{3 4}_{62} \text { Inferior cerebellar peduncle: } L 2 / L 3, F A / M O \text {. } \\
\mathbf{5 2}_{62} \text { Putamen/caudate T2*, anterior internal capsule, anterior thalamic radiation ICVF. } \\
\text { Haemoglobin, smoking, weight, meat intake. } \\
\mathbf{5 9}_{62} \text { Most white matter ISOVF/ICFV. Blood pressure, weight, smoking. }\end{array}$ & $\begin{array}{l}\text { VCAN } \\
\text { ZSCAN26 } \\
\text { ZSCAN23 } \\
\text { HLA-K } \\
\text { ZNF603P }\end{array}$ \\
\hline 56 & $\begin{array}{l}\text { rfMRI amplitudes } \\
\text { (sensory, motor and } \\
\text { cognitive). }\end{array}$ & $\begin{array}{l}\text { BMI, fat, weight, haemoglobin red } \\
\text { cell count, bone density, mobile } \\
\text { phone use, income. Blood pressure, } \\
\text { cardiac output, nervous feelings, } \\
\text { sleep duration. }\end{array}$ & $\begin{array}{l}\text { 1762 Cerebellar/subcortical rfMRI amplitude. BMI, haemoglobin, weight, fat. } \\
\mathbf{3 1}_{62} \text { Cognitive cortex rfMRI amplitude. Physical activity, blood pressure treatment, fat. } \\
\mathbf{3 3}_{62} \text { Sensory/motor/cerebellar/subcortical rfMRI amplitude/connectivity. Heart rate, } \\
\text { blood pressure, nervous feelings, night sleep duration, TV time, High SES / physical } \\
\text { activity (mobile phone use, daytime sleeping, physical activity, drive fast, income, risk } \\
\text { taking, number in house, number of sexual partners). } \\
\mathbf{4 1 6 2} \text { Visual rfMRI amplitude/connectivity. Age started wearing glasses. } \\
\mathbf{4 5}_{62} \text { Sensory/motor rfMRI amplitude/connectivity. Unenthusiastic, health-anxious, } \\
\text { depressed, lack of physical activity. }\end{array}$ & $\begin{array}{l}\text { PLCE1 } \\
\text { INPP5A } \\
\text { APOE }\end{array}$ \\
\hline 66 & Cortical thickness. & $\begin{array}{l}\text { BMI, weight, red cell count, head } \\
\text { bone density. }\end{array}$ & $\begin{array}{l}\text { 1562 Superior/medial frontal cortical thickness. } \\
\mathbf{2 3}_{62} \text { Precuneus/parietal. Birth weight. } \\
\mathbf{2 6}_{62} \text { Left lateral frontal. } \mathbf{2 8}_{62} \text { Right lateral frontal. } \\
\mathbf{2 9}_{62} \text { Sensory/motor (central superior). } \\
\mathbf{3 9}_{62} \text { Left post-central superior. Number of older siblings. } \\
\mathbf{4 3}_{62} \text { Right post-central superior. }\end{array}$ & $\begin{array}{l}\text { MACF1 } \\
\text { SLC39A8 / ZIP8 } \\
\text { PAFAH1B1 }\end{array}$ \\
\hline - & \multicolumn{4}{|c|}{ 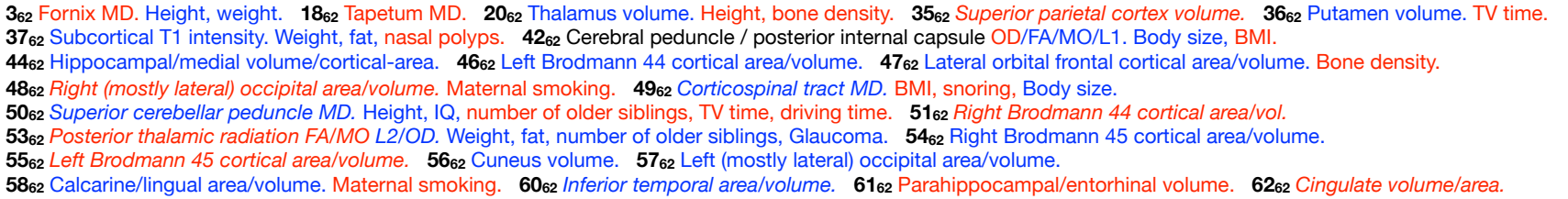 } \\
\hline
\end{tabular}

Table 2: Dominant imaging, non-imaging and genetic associations between brain-age delta from all mode-clusters and modes. The left side of the table focuses on the main patterns of associations with the 6 mode-clusters, while the right side also lists dominant associations with individual modes, grouped according to the mode-clusters. At the bottom are results from individual modes that do not have one clear associated mode-cluster. Red text signifies positive correlation with brain-age delta (meaning in general a detrimental factor with respect to aging), and blue indicates negative correlation (i.e., a positive causal factor and/or outcome with respect to aging). Where the all-in-one brain-age modelling has negative $\beta$, the signs of associations between delta and IDPs becomes the inverse of the original ICA IDP weight; in such cases the table makes this appropriate adjustment to text colour (such that the colour reflects the sign of assocation between delta and IDP, and not ICA weight), but we denote where this occurs by use of italics. Bold text indicates relatively stronger associations (in terms of strength of effects and/or number of related variables). Results included here are generally stronger than $-\log _{10} P>7$ for nIDPs (see Methods), and SNPs are listed only where replication succeeded. To help focus the descriptions of non-imaging variables, we largely list their associations with the partialled deltas; this therefore concentrates on unique variance in deltas. When working with partialled variables (or equivalently multiple regression), and when adjusting for some of the imaging confounds (such as head size, when considering volumetric measures), signs of associations can in some cases be non-trivial to interpret. 
bioRxiv preprint doi: https://doi.org/10.1101/802686; this version posted January 18,2020 . The copyright holder for this preprint (which was not certified by peer review) is the author/funder, who has granted bioRxiv a license to display the preprint in perpetuity. It is made available under aCC-BY 4.0 International license.
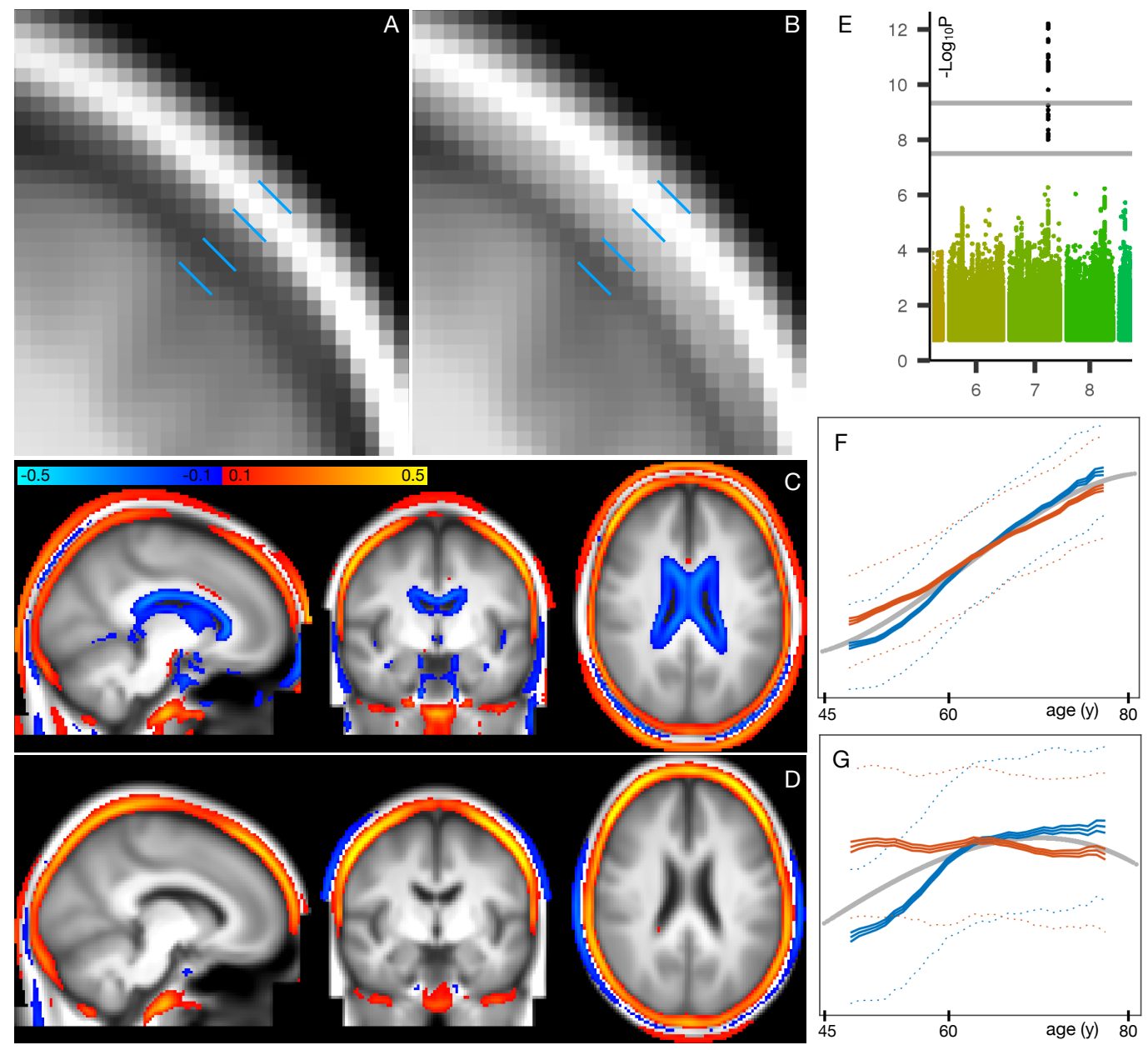

Figure 4: Spatial mapping of mode $\mathbf{1}_{62}$ onto original T1-weighted MRI data, along with genetic and age-dependent plots. A) A region-of-interest from the average T1-weighted structural image from the 1,000 subjects with the lowest delta values for this mode. The images have been linearly-aligned into standard (MNI152) template space, and have not been brain-extracted, so that non-brain tissues can be seen. The blue lines delimit 3 "layers" seen in cross-section; from the outside in, these are skin/fat outside the skull, the skull, and cerbro-spinal fluid outside of the brain. B) The equivalent average image from the 1,000 subjects with the highest delta values. There is no obvious geometric shift (e.g., of tissue boundaries), but the intensity values are clearly higher within the skull; this is reflecting increase in bone marrow fat with brain-age delta. C) The difference between the two average images (all images were first normalised to have a mean intensity of 1 ). D) The same difference of averages, but after regressing all confounds (including age) out of the voxelwise imaging data, and working with the partialled delta values for mode $1_{62}$; with this more focussed analysis, changes around the ventricle are no longer obvious, but the change in skull intensity remains. E) The one significant genetic association (on chromosome 7) for this mode. The lower grey line shows the standard single-phenotype threshold of 7.5; the upper line shows this after Bonferroni adjustment for multiple tests (modes). This significant association was also found in the replication dataset. F) The mean age curves for mode $1_{62}$ (as described in more detail in Methods and Fig. A.3). Females are shown in blue, males in orange; the $y$ axis is the unitless mode subject-weights (averaged across subjects with an averaging sliding window). The greatest rate of age-related change is in females, in the $10 \mathrm{y}$ following menopause. G) This pattern is even more striking in the partialled subject-weight curves (where other modes have first been regressed out of mode $1_{62}$.)

smoking and alcohol, as well as bone density (as measured separately from the MRI, using DEXA low-dose x-ray and also ultrasound). This bone density association is strong, reaching $r=0.43$ in females and 0.27 in males. The greater bone density loss in females is likely to be associated with menopause. Firstly, this mode is significantly associated with age-at-menopause (a non-imaging variable in UK Biobank, with average age-at-menopause being 50y). More generally, there is a large amount of literature showing that bone density loss is specifically accelerated in the 10 years after menopause O'Flaherty, 2000; this exactly matches the sex-specific pattern of change in females seen in this mode (Fig. 4 F, G). 
Fig. $4 \mathrm{~A}, \mathrm{~B}$ shows the increase in T1-weighted intensity within the skull, associated with this mode. This is reflecting an increase in bone marrow fat with increasing brain-age delta. This, together with the above nIDP associations with bone density loss in this mode, is consistent with literature regarding decreasing bone density and increasing marrow in aging Cordes et al., 2016. Bone density reduction has not just been reported in normal aging, but has also been linked to early Alzheimer's disease, independent of age, sex, habitual physical activity, smoking, depression and estrogen replacement status [Loskutova et al., 2009].

These results are consistent with the one strong genetic association with mode $1_{62}$ (Fig. 4F); lead SNP rs3801383 (whose PHEWAS results are dominated by bone density associations - see Methods) lies within the span of FAM3C, but also is in LD with SNPs spanning across to genes WNT16 and CPED1 [Chesi et al., 2015, Moverare-Skrtic et al., 2015. FAM3C is associated (in UK Biobank genetic data http://big.stats.ox.ac.uk) with bone density loss and bone fractures, but has also been linked directly to Alzheimer's disease through impact on brain amyloid [iu et al., 2016.

Mode-cluster $\mathbf{3}_{6}$ singles out IDPs representing T1-weighted intensity contrast between white and grey matter (across the greywhite border). Although mode-clusters $1_{6}$ and $2_{6}$ related to weight, they were essentially driven by non-fat mass; here, however, mode-cluster $3_{6}$ mainly relates to measures of fat mass and fat percentages across the body, as well as blood haemoglobin measures.

In line with these non-imaging correlations, one strong genetic association was found for a SNP (rs12133923), an eQTL of CREB3L4 in the basal ganglia and of CRTC2, SLC27A3 and S100A16 in the cerebellum. CREB3L4, which regulates adipogenesis, has for instance been recently shown to have a critical role in metabolic phenotypes (weight gain, impaired glucose tolerance and decreased insulin sensitivity) [Kim et al., 2015]. CRTC2 plays a role in lipid metabolism, and SLC27A3, which encodes fatty acid transport protein, is involved in the developmental stage of the central nervous system Maekawa et al., 2015. Taken altogether, it is therefore likely that the marked, widespread change of cortical contrast with aging witnessed here (and in several previous studies) is strongly related to the fatty, lipid-rich myelin Salat et al., 2009, Vidal-Piñeiro et al., 2016 Lewis et al., 2018.

Of note, mode-cluster $3_{6}$ was also strongly associated with other SNPs, amongst them one (rs1044595) in an exon of STX6, and in high LD with a SNP associated with tauopathy progressive supranuclear palsy[Höglinger et al., 2011], and correlated in the UK Biobank participants with hormonal replacement treatment. Another hit (rs6442411), an eQTL of WNT7A, which regulates angiogenesis, neurogenesis and axon morphogenesis, was associated in the UK Biobank population with height and trunk mass. One SNP, rs541397865, was found in an intron of CD82; this regulates the migration of oligodendrocytes, which are responsible for axonal myelination. We also found a genetic association with rs10052710, a SNP in an intron of VCAN, and in high LD with a previous hit we had found strongly associated with diffusion measures across the entire white matter [Elliott et al., 2018]. These additional associations further point to the driving contribution of myelin in the aging-related modulation of grey/white-matter contrast.

Mode-cluster $\mathbf{4}_{6}$ is strongly linked to modifiable risk factors: high blood pressure, vascular and heart problems, and associated with taking ramipril (a treatment against high blood pressure and heart failure). It was also associated with a number of illnesses and treatments, including multiple sclerosis. Mode-cluster $4_{6}$ is characterised by diffusion measures of mostly frontal white matter (anterior corona radiata and, overlapping in the frontal lobe, the inferior fronto-occipital fasciculus), and was also associated with general reaction time. The subject-weights are strongly age-dependent (as are all mode-clusters); however, they have very little age dependence after partialling out other mode-clusters; this means that the above factors interact in a manner that is largely age-independent.

Genetic associations included again a SNP in an intron of VCAN (rs17205972, in high LD with the VCAN SNP associated with mode-cluster 36 , and reported in [Elliott et al., 2018). Additionally there was association with SNP rs3129787, an eQTL in the brain of ZSCAN26 and ZSCAN23 (in the cortex and cerebellum), HLA-K (cortex), and ZNF603P (basal ganglia, cortex, hypothalamus, cerebellum), a pseudogene whose expression in the brain has been recently observed to be associated with schizophrenia and affective disorders Bhalala et al., 2018]. The latter SNP was also highly correlated in the UK Biobank participants with health issues including coeliac/malabsorption disease, blood pressure, taking insulin and hyperthyroidism, as well as with measures of lung function.

Mode-cluster $\mathbf{5}_{6}$ shows a modest deceleration of aging-rate with increasing age, particularly with respect to its unique (partialled) variance (Fig A.9). It involves just the amplitudes of resting-state fluctuations, covering most of the brain; some of the associated modes also show rfMRI connection-strength involvement, but that may be an indirect result of the amplitude changes. Mode-cluster $5_{6}$ demonstrated strong correlations with non-imaging variables similar to mode-cluster $3_{6}$ : weight, fat mass and percentage, red blood cell count and haemoglobin. It also was associated with blood pressure, cardiac output and bone density, along with sleep duration, nervous feelings and several markers of socio-economic status (SES). 
Mode-cluster 5 was strongly associated with several SNP clusters, having relevant correlations in the UK Biobank population: rs7766042 with snoring; rs2273622 with high blood pressure, migraine and headache, taking pain relief, vascular and heart problems; and rs2274224 with weight, (fat-free, and fat) mass, fat percentage and blood pressure (including taking amlodipine). This latter SNP is in an exon (missense) of PLCE1, as also seen in [Elliott et al., 2018] and [Hübel et al., 2019], another recent UK Biobank study on body fat percentage. The strongest GWAS hit is rs 4497325 , and for the associated mode $45_{62}$, the peak SNP is (the immediately-neighbouring) rs7096828; this is an eQTL of INPP5A, which is involved in DNA methylation in neurons, associated with aging and depression Gasparoni et al., 2018.

Finally, a genetic association was found with rs429358, the SNP that determines whether the APOE allele is $\epsilon 4$ or not. This is a major locus associated with Alzheimer's disease and mild cognitive impairment, and also with dementia with Lewy bodies, age at onset of symptoms in Parkinson's disease, insomnia, brain amyloid deposition and neurofibrillary tangles, inflammation, $\mathrm{HDL} / \mathrm{LDL}$ cholesterol and triglycerides levels, physical activity and blood protein levels, parental longevity, and macular degeneration. In the UK Biobank participants http://big.stats.ox.ac.uk this SNP also correlated with Alzheimer's disease in father/mother/siblings, LDL/cholesterol levels (and taking cholesterol-lowering medication), omega6, triglycerides, diabetes in the mother, weight and fat mass, with heart disease and with the mother's and father's age at death, amongst many other variables.

Despite being associated with SES, mental health markers, functional MRI amplitude fluctuations, and SNPs involved in cognitive decline, there were no direct associations between this mode-cluster (or its associated modes) and cognitive test scores. In the case of the IDPs, this may well mean that the changes seen are non-neural effects (e.g., cardiovascular causes of changes in the BOLD amplitude), and that any associated cognitive effects are caused by ongoing damage and not seen until later in life than the majority of the samples (imaged subjects) here. Even in the mode covering cognitive brain regions $\left(31_{62}\right)$, the set of nIDPs is dominated by exercise/activity measures and not cognitive test scores. The link between SES and fMRI activity levels seen in Miller et al., 2016] (Fig. 7C) may now be explained; here, mode $41_{62}$ links amplitude of rfMRI fluctuations in visual cortex to age when started using glasses (and indeed we looked at age subgroups to confirm that this association is driven by those subjects who started wearing glasses while younger than $30 \mathrm{y}$ ).

Mode-cluster $\mathbf{6}_{6}$ was entirely composed of grey matter thickness IDPs, mainly in the prefrontal areas, as well as higher-order parietal and temporal regions. It correlated with non-imaging variables of weight, red blood cells and head bone density. This mode-cluster was age-dependent, but its unique (partialled) variance was only weakly so.

We found 3 genetic associations with Cluster 6. The first, rs682571, is in an intron of MACF1, which has been shown recently to regulate the migration of pyramidal neurons and cortical GABAergic interneurons Ka et al., 2014 Ka et al., 2017. This SNP also correlated in the UK Biobank population with several measures of body fat. Another hit, rs13107325, is in an exon (missense) of SLC39A8 (ZIP8), the same SNP reported in our GWAS-IDP study [Elliott et al., 2018 to be associated with subcortical and cerebellar volume and susceptibility. This has also been found in other GWAS studies (many based primarily on UK Biobank data), including those looking at medication use, tobacco and alcohol consumption, cholesterol, body fat, adiposity, osteoarthritis, red blood cell, blood pressure, sleep duration, risk taking, intelligence/math ability/cognitive function and schizophrenia. A final SNP, rs7219015, was found in an intron of PAFAH1B1 that, when mutated, leads to lissencephaly. It is also found to correlate with tiredness in UK Biobank Deary et al., 2018.

\section{Discussion}

Here we aimed to study how multiple, biologically distinct, modes of population variation in brain structure and function reflect different aspects of the aging brain. We investigated the modes' distinct associations with genetics, life factors and biological body measures, in the context of the modelling of brain age and brain-age delta - a measure of whether subjects' brains appear to be aging faster or slower than the population average.

To study these multiple modes, we used brain imaging data from six different imaging modalities spanning many different aspects of brain structure and function, from 21,407 subjects, from a single, highly homogeneous, study. All imaging data was first reduced to 3,913 IDPs (imaging-derived phenotypes - summary measures of brain structure and function) from across the different modalities. However, rather than studying aging in different individual IDPs, we identified latent factors of population covariation using unsupervised learning, to provide a more compact, lower-noise representation of the population data, and focussing only on population modes showing extremely high split-half reproducibility.

All imaging data (and the same set of IDPs) used for our work here are available from UK Biobank, as is all code used for 
the core UK Biobank processing, and new code generated for this work is also freely available. Therefore, for data from other (non-UK Biobank) studies, the full code is available for deriving the exact same set of IDPs, as long as the same imaging modalities are acquired. How well harmonised those IDPs would be with UK Biobank IDPs would of course be a "sliding-scale", dependant on how similar the MRI scanner hardware, scanner software and protocol were to those in UK Biobank. Similarly, how similar any derived brain modes would be to those that we report here would likewise be a sliding-scale, dependant on how similar the data characteristics (and subject group demographics) were.

Previous work showing more than a single pattern of brain aging includes [Groves et al., 2012, where we used voxel-level multimodal independent component analysis (ICA) applied to data from 484 subjects, to generate multiple population modes, several of which showed age dependence (including early-life development). However, this data spanned almost the entire human age range (8-85y), with data from just two imaging modalities, and hence did not identify a large number of distinct modes relating to older-age aging. In the same year, a study of early-life development and maturation (885 subjects, 3-20y) used 3 imaging modalities to generate 231 distinct imaging features [Brown et al., 2012. The features were then grouped into different subsets by hand, and the age dependence of each subset (and also of many of the features on their own) was studied. Similarly, Vinke et al., 2018 included data from several modalities, and studied aging trajectories in different measures from different modalities, but did not go as far as brain age (or brain-age delta) modelling, or attempt to identify latent modes of aging. Several modalities were also used in Richard et al., 2018, with 11 groups of distinct measures used to form 11 estimates of brain age, each of which was then separately investigated for cognitive associations; one central methodological distinction to the work presented here is that the 11 models were hand curated according to different types of features from different modalities, as opposed to (in our case) pooling all modalities' features together before using data-driven decomposition (ICA) to identify distinct aging modes that could naturally span across feature types and modalities. In contrast, Kessler et al., 2016 used single-modality features (resting fMRI edge strengths) fed into ICA to identify multiple modes of early-life maturation. Finally, Kaufmann et al., 2019] used a single imaging modality (T1-weighted structural images) from 45,000 subjects pooled from 40 studies, to investigate the relationship between brain aging and several diseases. Brain-age prediction was trained from whole-brain analysis of the structural data, and also seven atlas-defined regional subsets were used to retrain the predictions. The different regional brain-age delta estimates showed varying associations with disease. However, as with our all-in-one predictions and also Ning et al., 2018, direct GWAS of the delta estimates showed virtually no significant assocation, even with these high subject numbers.

We suggest that there is value in considering multiple, multimodal, brain aging modes separately; for example, while our single all-in-one modelling of brain-age delta had no significant genetic influence, many of the individual modes had significant, rich and biologically interpretable genetic influence. We also found rich patterns of significant associations with non-imaging non-genetic variables, including: biological measures (bone density, body size and fat measures, metabolic and cardiovascular function, blood pressure, haemoglobin, age at menopause); life factors (alcohol, smoking, maternal smoking, physical activity, number of siblings, sleep duration, many markers of socio-economic status); cognitive test scores (processing speed, IQ); mental health (anxiety, depression); and disease (diabetes, multiple sclerosis). To help focus our reporting of these non-imaging variables, we largely considered their associations with the partialled deltas, i.e., concentrating on unique variance in each mode's delta. However, doing this is not mandated where the non-imaging variables (e.g., blood pressure) or genetics are more likely to be causal factors than caused, in which cases, the (in general less conservative) correlations with non-partialled deltas can be more appropriate.

The multiple modes of brain aging involved all imaging modalities, in a range of different patterns. Some modes spanned multiple modalities, while others were more focussed, primarily reflecting within-modality patterns. Measures of brain structure and function included: volumes of grey and white tissues and structures (e.g., ventricles, thalamus, hippocampus); intensity contrast between grey and white matter; microstructural measures in white matter tracts (diffusivity, anisotropy); amplitude of spontaneous fluctuations in grey matter fMRI amplitude, and functional connectivity between regions; volume of lesions in white matter; and changes in susceptibility-weighted contrast (likely reflecting iron deposition) in subcortical structures. Of course, while all imaging modalities did show some involvement in the brain-aging modes, it is not the case that all are equally valuable, both in terms of reflecting the true underlying biology of brain aging, or in terms of the reliability with which they could be estimated from the UK Biobank imaging data. For example, the fMRI IDPs in general seem noisier than structural IDPs, as seen in our previous GWAS results [Elliott et al., 2018]; however, it would be incorrect to conclude that the noisier IDPs contain no useful information, and indeed we showed in the same study that a data-driven reduction of hundreds of individual fMRI IDPs to a small number of latent factors did show significant genetic association. Similarly, here, the fMRI-based modes were not strongly dominant, but were nevertheless reproducible (see Methods). Additionally, having included the "relatively noisy" fMRI IDPs did not damage estimation of more structural modes: if we exclude fMRI IDPs from all analysis, and rerun the mode-cluster estimation, the 5 mode-clusters not dominated by fMRI IDPs were still found (in all cases having high correlation, $r>0.9$, with their original estimation). 

aCC-BY 4.0 International license.

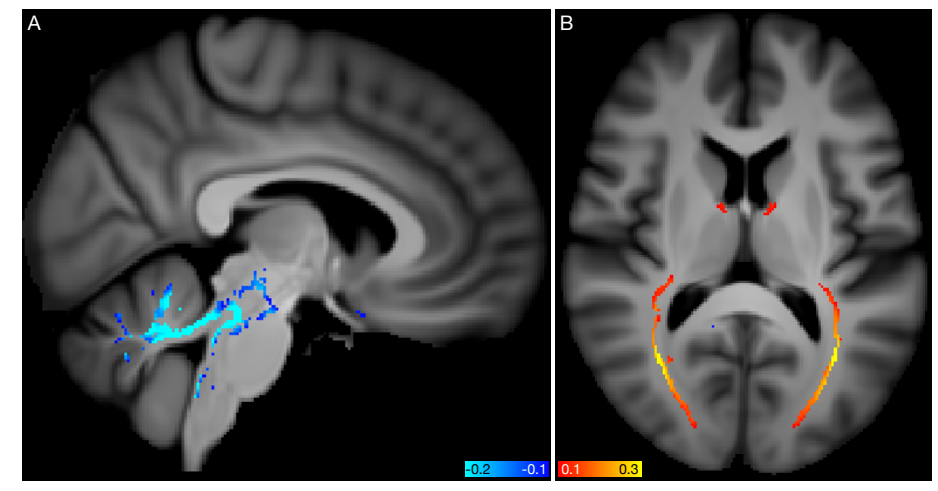

Figure 5: Spatial mapping of modes $\mathbf{5 0}_{62}$ and $\mathbf{5 3}_{\mathbf{6 2}}$ from the diffusion MRI data. A) Voxelwise correlation of the partialled brain-age delta values (one per subject) from mode $\mathbf{5 0}_{\mathbf{6 2}}$, into the $\mathrm{dMRI}$ MD (mean diffusivity) data. The colour overlay shows correlation $r$ values, thresholded at a magnitude of 0.1 . B) Voxelwise correlation of the partialled brain-age delta from mode $\mathbf{5 3} \mathbf{6 2}_{\mathbf{6 2}}$, into the dMRI FA (fractional anisotropy) data.

Although there is a good deal of literature relating patterns of normal brain aging to some diseases (including our results discussed above), one should not assume that all diseases display patterns identical to accelerated normal brain aging. This does not mean that the study of normal brain aging would not be of value in such diseases; indeed, thorough characterisation of normal brain aging could well help disentangle disease effects from (non-disease) aging effects in the subjects with disease. Additionally, identification of latent factors of population variation (such as carried out here) may help in the discovery of distinct disease sub-groups.

All modes' subject-weight vectors are oriented (by definition) to increase with age. However, with respect to their unique signal (obtained by regressing out all other modes from any given mode), a small number of modes are negatively correlated with age, as described in Methods and shown in Fig. A.2 Two examples are visualised in Fig. 5 Mode $53_{62}$ involves changes in white matter fibre organisation in the posterior thalamic radiation (also known as the optic radiation, and connecting to visual cortex), and was associated with glaucoma, as previously reported [Wang et al., 2018]. Mode $50_{62}$ involves changes in white matter diffusivity in the superior cerebellar peduncle, and was associated with IQ and several markers of socio-economic status. In such cases, where a mode's unique variance is contributing to reducing (and not increasing) brain age, one possible interpretation is that the mode represents cognitive reserve, i.e., working against the general pattern of age-related decline (indeed, aspects of socio-economic status are frequently used as proxies for cognitive reserve). The primary goal of this work is the decomposition of aging effects in the brain into multiple modes, which would ideally be biologically distinct and interpretable. It is unsurprising that, by incorporating all modes (and hence all IDPs), the all-in-one brain-age modelling achieves higher accuracy in age prediction; however, this is achieved at the expense of diluting associations, as seen here with the genetics. It nevertheless could be the case that deeper consideration of how the modes interact with each other to achieve optimal integrated modelling (for example, considering their "partialled" regression parameters in depth) may bring new understanding about brain aging. For example, this could shed new light on how different external causal factors and distinct brain aging responses to these interact with each other.

A major premise behind the modelling of multiple brain-aging modes is that each mode in each subject has a distinct delta: for a given subject, the different modes are "aging" differently. Although the common approach in the brain-aging literature is to estimate a delta (or brain age gap) by subtracting actual age from estimated brain age, this has the potential weakness of assuming that this offset would be constant for a given subject, as the subject gets older. For example, this assumption is implicit when looking for genetic associations, as one would like to be finding associations with an age-independent marker of relative brain health. However, it may be more likely that (for example) a given subject's brain is aging faster than the population average in terms of a distinct aging rate, implying that their delta would be increasing over time (and not therefore being a constant offset relative to their age). Indeed, our results show that there is evidence for this being a more appropriate model of brain aging (Fig A.15 shows several modes where the variance of delta increases with age, and a few where it decreases). Unfortunately, the two models can be hard to distinguish, particularly at the level of individual subjects, when given only single-time-point (cross-sectional) data. For example, it may be hard to disambiguate whether total brain volume is different than the population average because of aging effects, or because the subject had a larger/smaller brain at "baseline" (before age-related decline began). This is similar to the distinction between "shallow" vs. "lagged" early-life maturation investigated by [Kessler et al., 2016]. Naturally some preprocessing helps ameliorate this, for example, normalisation of brain volume by 
head size. However, it is still the case that longitudinal data, and more advanced modelling, may result in more sensitive and meaningful study of brain aging in future. Notably, UK Biobank has now started re-imaging 10,000 of the 100,000 subjects, with an average scan-rescan interval of about 2 years. Raw and preprocessed data from almost 1,500 of these rescanned subjects will be released in early 2020. Future work on brain-age modelling can hope to take advantage of the ever-increasing size and richness of such datasets, to enable better understanding of the aging brain in health and disease.

\section{Methods}

\subsection{Data and preprocessing}

We used data from 21,407 participants in UK Biobank, 53\% female, aged 40-69y at time of recruitment and 45-80y at time of imaging. As described in detail in [Miller et al., 2016], the UK Biobank data includes 6 MRI modalities: T1-weighted and T2-weighted-FLAIR (Fluid-Attenuated Inversion Recovery) structural images, susceptibility-weighted MRI (swMRI), diffusion MRI (dMRI), task functional MRI (tfMRI) and resting-state functional MRI (rfMRI).

We (and colleagues) have developed and applied an automated image processing pipeline on behalf of UK Biobank AAlfaro-Almagro et al., 2018 https://www.fmrib.ox.ac.uk/ukbiobank/fbp This removes artefacts and renders images comparable across modalities and participants; it also generates thousands of image-derived phenotypes (IDPs), distinct measures of brain structure and function. Here we used 3,913 IDPs available from UK Biobank, spanning a range of structural, diffusion and fMRI summary measures (as described in the central UK Biobank brain imaging documentation http://biobank.ctsu.ox.ac.uk/showcase/showcase/docs/brain_mri.pdf and listed in full in a spreadsheet available at https://www.fmrib.ox.ac.uk/ukbiobank/BrainAgingModes).

Code for all processing in this paper is freely available (see Section 5). Each IDP's $N_{\text {subjects }} \times 1$ data vector had outliers removed (set to missing, with outliers determined by being greater than 6 times the median absolute deviation from the median); the vector was then quantile normalised Miller et al., 2016, resulting in each IDP's data vector being Gaussian-distributed, with mean zero, standard deviation one. We then discarded subjects where 50 or more IDPs were missing (for any reason, which could be due to: data acquisition incompleteness; data quality problems as described in [Alfaro-Almagro et al., 2018; or the above-described outlier removal), leaving 18,707 subjects ( $54 \%$ female). The small amounts of remaining missing data were replaced with close-to-zero values (random signal of standard-deviation 0.01). This resulted in an IDP data matrix $W$ of size $18,707 \times 3,913$.

Confounds were removed from the data as carried out in [Elliott et al., 2018] (except that age-dependent confounds were not removed from $W$ ). This includes confounds for: head size, sex, head motion during functional MRI, scanner table position, imaging centre and scan-date-related slow drifts.

In applications with a specific disease of focus, it is common to generate a model such as brain-age-estimation based on healthy subjects and then apply it to both healthy and disease subjects. However, here (and in UK Biobank in general) there is no one specific disease focus, with all diseases being of potential interest, and with the imaged population being largely healthy at the time of imaging. The fractions of imaged subjects having specific existent diagnoses are low (for example, with less than $10 \%$ having mental health or neurological diagnoses, and none having gross anatomical pathology according to the processing pipeline QC [Alfaro-Almagro et al., 2018]). We therefore did not exclude individual subjects from the modelling here.

\subsection{Estimation of multiple population modes of brain aging}

We then applied independent component analysis (ICA), using the FastICA algorithm Hyvärinen, 1999. ICA decomposes a data matrix into multiple factors that are statistically independent of each other with respect to one of the data matrix dimensions (the input data matrix is $W$, meaning that the data dimensions are subjects and IDPs). This generates multiple independent modes of population covariance (patterns of IDPs that co-vary together across subjects).

In order to help focus this data-driven decomposition on age-related population modes, both with respect to the pre-ICA dimensionality reduction (achieved using PCA - principal component analysis) and the core ICA unmixing, each IDP vector $W_{i}$ (after normalisation as described above) was rescaled by an age-related factor of $\left(0.1+a b s\left(\operatorname{corr}\left(a g e, W_{i}\right)\right)\right)$, before PCA+ICA was applied. 
ICA requires the estimated (output) components to be non-Gaussian in their distributions, and our data matrix $\mathrm{W}$ is more highly non-Gaussian in the IDP dimension than in the subject dimension (which is largely Gaussian for most IDPs, even before quantile-normalisation). We therefore appply ICA to estimate modes of independent IDP weights. Each ICA component therefore comprises a mode of population covariation described by two vectors: the "ICA source vector", spanning all IDPs, with one (signed) scalar weight value per IDP; and the subject-weights vector, with one (signed) scalar weight value per subject. The rank-1 outer product of these two vectors comprises this mode's contribution to the full original data matrix. IDP-weightvectors are statistically independent of each other (by definition, according to the ICA algorithm) and hence also orthogonal, whereas the subject-weight-vectors are only restricted to being non-co-linear (and indeed below we utilise their correlations with each other to help identify clusters of modes).

Estimation of association of a given mode with age or non-imaging variables (such as cognitive test scores and physical body measures) can proceed simply by correlating/regressing the subject-weights vector against any relevant non-imaging $N_{\text {subjects }} \times 1$ vector. As described above, all modes have distinct (from each other) subject-weights-vectors and IDP-weights-vectors, and hence are distinct modes of population variation. Note that the ICA algorithm will always produce the requested number of modes, and as such the statistical robustness of identified modes requires some form of test, such as the reproducibility testing described below.

A major controlling parameter in an ICA decomposition of a data matrix is the number of components it is asked to estimate - i.e., how fine-grained the "clustering" output should be. It is common to specify just one controlling parameter when running FastICA, that being the initial PCA dimensionality reduction. ICA would then output the same number of components. However, it is also possible to control the PCA dimensionality, and separately determine which ICA output components to keep. Our general approach (detailed below) was to maximise both dimensionalities separately, in order to obtain the richest possible description of multiple population modes. However, this needs to be done with the constraint that reported modes are statistically robust (i.e., avoiding over-fitting).

Therefore, starting from 3,913 columns (IDPs) in $W$, we ran PCA and ICA at dimensionalities from 60 to 150, evaluating each with respect to a metric of split-half reproducibility (all code for this is available, as described above). For each PCA dimensionality reduction, this test of reproducibility applies the following procedure: ICA is run three times - first with all data, and then twice on randomly-split-halves of the data; the components from the two split-half runs are then ordered according to best-match (via the Hungarian greedy-pairing algorithm) to the all-data ICA run; correlation between the split-half paired ICA components' source (IDP) vectors was estimated, and only extremely similar components $(r>0.9$, see below for estimation of the associated statistical significance) were retained; all the above steps were run 10 times (each with a different split-half-subjects randomisation) and averaged together to give the reproducibility test-statistic - the number of reproducible components estimable by the current dimensionality.

The PCA dimensionality resulting in the largest number of highly reproducible components was found to be 128 , and from this, 62 ICA components were highly reproducible. Finally, ICA was rerun with this PCA dimensionality 30 times, each time with random split-half-subjects, and the most robust run (in terms of reproducibility) was then utilised, resulting in the final set of 62 ICA components.

As a simple highly conservative test of significance, we computed null correlations between an "IDP-weight vector" of random noise of 62 samples (the minimum possible degrees-of-freedom, and hence the most conservative test) and 128 other random vectors, taking the maximum correlation magnitude across all 128 , and then building up the null distribution of this maximum across 1 million random null tests. The maximum across all 1 million only reached $|r|=0.68$ (90th percentile $|r|=0.41$ ), whereas we are only keeping modes with split-half reproducibility $|r|>0.9$. We can therefore be confident that the final components are robustly present with a significance of at least $P<10^{-6}$ (and probably much greater).

As a second test of significance of the overall data-driven modelling, including the age-weighting of inputs to the PCA+ICA, we applied the following null evaluation. We used a random vector instead of age to carry out the IDP weighting, ran ICA at dimensionality of 128 , and correlated all 128 resulting subject-weight-vectors with the random vector, recording the maximum correlation magnitude across all 128 (and by doing so making this more conservative than by testing just 62 modes chosen through split-half reproducibility). From 100 random repeats of this test, the maximum absolute correlation (across 100 repeats and 128 ICA modes) was just $|r|=0.032$ (to be compared against the age correlations shown in Fig. A.2P).

A given ICA component is unchanged in its modelling of the input data if the sign of both the subject-weights-vector and the IDP-weights-vector are inverted (as these two inversions cancel each other out - the initial sign of each is arbitrary, as with PCA). Hence we oriented the 62 modes of population variability so that their subject-weights-vectors were all positively correlated with age, in order for simplicity of later interpretation. 
We next investigated whether the 62 modes of brain aging could be arranged in fairly clean clusters having similar patterns of aging; if so, this could aid in simplifying interpretation of the modes. Fig. A.1 shows hierarchical clustering of the correlation matrix of subject-weight-vectors. The reasonably strong diagonal-block-structure suggests that a lower-dimensional clustering could be a useful way to help simplify the interpretation of the 62 modes of brain aging. Therefore, in order to carry out a lowerdimensional analysis, we re-ran the ICA, this time on PCA dimensionalities running from 2 to 50 (from the same IDPs matrix that was fed into the higher-dimensional mode estimation above). We evaluated objectively which dimensionality provided the cleanest clustering of the 62 modes, by optimising the following cost function: We estimated the correlation matrix of 62 modes' subject-weight vectors with each low-dimensional ICA set of subject-weight vectors, took the magnitude of this, sorted each column (spanning the low-dimensional analysis), subtracted the second-strongest correlation from the first, and summed this over columns (the high-dimensional components). This cost function therefore describes how cleanly each high-dimensional mode is associated with just a single low-dimensional component. We found that the optimal lower dimensionality was 6 .

As well as being sign-oriented to positively correlate with age, the modes (from both 62 and 6 dimensionalities) were ordered (numbered) according to decreasing correlation with age, again for convenience of interpretability and with no loss of generality in the modelling. We refer to the higher-dimensional modes of aging via their (ordered) number with subscript 62 (e.g., "brain aging mode $2_{62}$ "), and lower-dimensional mode-clusters via their number with subscript 6 (e.g., "brain aging mode-cluster $3_{6}$ "). Fig. A.1 3 shows the correlation matrix between subject-weight-vectors from the two dimensionalities, with the fairly clear clustering visible (i.e., most of the 62 modes are strongly associated with at most one of the 6 mode-clusters).

\subsection{Brain-age visualisation and sex-separated aging curves}

We used the estimated population modes to model brain aging, following the general regression-based approaches laid out in [Smith et al., 2019].

For simple visualisation of each mode's overall age dependence, we utilise the simple "switched" model, where imaging measures are characterised as a function of age. We used an age model with linear, quadratic and cubic powers of age, to fit to each mode's subject-weights-vector. The fitted age curves for all 62 modes are shown in Fig. 1 as well as the raw data (scatterplot points, one per subject) and fitted curves for 2 example modes. By definition (see above), all modes have positive age correlation, although for some modes these positive coefficients are close to zero. Fig. A.1C shows the equivalent fitted age curves for the 6 mode-clusters. Fig. A.2A shows the ratio of the standard deviation explained by the mean-age-dependent-curves to the standard deviation of the data (the mode subject-weights). There is a continuous distribution of ratio values, from above 0.6 in the lowest-numbered modes, through to almost zero for the highest-numbered modes (though all of the mode-clusters are above 0.3). (Significance testing on strength of age dependence is reported below).

Figs. A.3 A.9 show, for each mode, sex-separated aging curves, and also the aging curves for the unique variance captured by each mode. For the latter, the subject-weights-vector for each mode is "partialled" - i.e., has all other modes' subject-weightsvectors regressed out, before re-fitting the average age curves for visualisation in the figures. For these sex-separated aging curves, sex-separated subject-weight-vectors were first estimated, by multiplying the ICA IDP weights matrix into a version of the original data matrix that had all confounds removed as before, but this time without including sex as one of the confounds. Averaged age-curves were then generated; for these visualisations, sex-separated age curve fitting was carried out in a more model-free way than the parametric (cubic) age model used for our more quantitative analyses. Specifically, for the purposes of showing the data in a more raw form, we simply use sliding windows of width $5 y$ to average (sex-separated) data points around each $1 y$ age bin centre (although averages of the two sex-separated curves are visibly highly consistent with the cubic average age model shown underneath in grey). For the majority of modes, the two sexes have highly similar age curves, but for some (e.g., mode $1_{62}$ ), there are strong differences.

As one would expect, the age dependence is less strong in the partialled modes, as each has a large amount of shared variance regressed out. Some even show negative overall age dependence after partialling (e.g., mode $22_{62}$ ).

\subsection{Brain-age delta modelling}

For our quantitative modelling of brain-age delta (estimated brain-age minus actual age), we use the common approach of modelling age as a function of imaging features (as opposed to the other way round as above), combined with the second step from [Smith et al., 2019], which removes age-related bias in the brain-age delta. Hence, for the first step, one would model

$$
Y=X \beta_{1}-\delta_{1},
$$


where $Y$ is age, $X$ is the modes' subject-weights matrix (size $\left.N_{\text {subjects }} \times N_{\text {modes }}\right), \beta_{1}$ is the $\left(N_{\text {modes }} \times 1\right)$ vector of regression parameters, and $\delta_{1}$ the initial estimate of brain-age delta. The above produces a $\delta$ that is orthogonal to $X$ (the imaging measures) rather than $Y$ (age). Thus, we can think of the first stage residuals, $\delta_{1}$, as the aspects of age that cannot be accounted for by the imaging measures. The second stage of modelling aims to refine this model by identifying aspects of this first-stage $\delta_{1}$ that cannot be accounted for by age terms or confounds. Note that this stage explicitly forces $\delta_{2}$ to be orthogonal to all of the components in $Y_{2}$, including age:

$$
\delta_{1}=Y_{2} \beta_{2}+\delta_{2},
$$

where the regression matrix $Y_{2}$ includes not just linear, quadratic and cubic age terms, but also the other confound variables. One can equivalently view the first step above as a sum over modes:

$$
Y=\sum_{i}\left(X_{i} \beta_{1 i}-\delta_{1 i}\right)
$$

where we have separated out the contributions to the modelling from each mode, along with breaking down the delta into a delta vector per mode. The $\beta$ regression parameters remain determined by the standard multiple regression inversion, $\beta_{1}=\left(X^{\prime} X\right)^{-1} X^{\prime} Y$, and each $\delta_{1 i}$ is estimated simply as $X_{i} \beta_{1 i}-a Y$. Here $a$ is an arbitrary scaling (e.g., $1 / N_{\text {modes }}$ ) whose value is not important because the term $a Y$ will be removed by the second step that regresses out age and confounds. One can then keep the second step deltas also separated:

$$
\delta_{1 i}=Y_{2} \beta_{2 i}+\delta_{2 i},
$$

The original $\delta_{1}$ is the sum of the individual modes' $\delta_{1 i}$ vectors, and $\delta_{2}$ is the sum of all modes' $\delta_{2 i}$ vectors. By separating out each mode's contribution to the overall brain aging delta, and by doing so in the context of the modelling being an "all-in-one" multivariate model (multiple regression using all modes' subject-weights vectors), we are able to then go on to study how the different modes' brain-aging are distinct from each other, as well as how they combine to give an overall best-estimate of brain age. The combined modelling across all modes (summed $\delta_{2 i}$ ) results in a mean absolute "error" of $2.9 \mathrm{y}$.

As with the partialled subject-weight-vectors described above, we also generate partialled versions of the modes' deltas; for each mode's $\delta_{2 i}$, we regress out all of the others. We can then, for example, correlate these partialled delta estimates with non-imaging variables in order to find associations with the unique variance in each mode's brain-aging delta.

In Fig. A.2 $3, C$ we show the standard deviation (variation across subjects) associated with the individual modes' brain-age modelling from step $1\left(X_{i} \beta_{1 i}\right)$, the deltas after step 2 , and the partialled deltas. There is not a qualitative difference between the 3 curves, because the $\beta$ regression parameters are driven by the unique variance components of the original modes' subjectweight-curves. There is not (expected to be) a simple relationship between the original strength of age dependence for a given mode, and the age dependence in its unique variance; this also explains why the curves are not monotonically decreasing (as they clearly are, by definition, in the univariate analyses shown in Fig. A.2A).

In Fig. A.2 D, E we show related information - statistics from the multiple regression in the age modelling first step (as well as the simple univariate correlation between individual modes' subject-weight-vectors and age, for reference). The regression $\beta$ values vary highly from mode to mode (as mentioned above), driven by the unique variance in each mode. Several modes have negative $\beta$ weights, meaning that their unique variance is negatively associated with age, even though their original correlation with age was (by definition) positive. Two modes $\left(22_{62}\right.$ and $50_{62}$ ) have quite strongly negative $\beta$ (more negative than -0.5 ).

\subsection{Non-additive brain-age delta estimation}

Following the approach outlined in [Smith et al., 2019], we estimated the extent to which the scale (size) of delta changes across the age range present in the UK Biobank data. This is a distinct model from those outlined above, which treat delta as additive to age (to form brain age), and hence being constant in overall scale (as a function of aging). This would represent not a simple shift in brain age, but potentially (e.g.) something like an acceleration in aging (delta gets bigger with age). Of course, with a limited range of ages, such a scaling term might be effectively captured with a purely additive term, so this modelling is really asking whether our data show evidence for a scaling effect, rather than making a strong statement about the form deltas take over the entire age range.

The results are shown in Fig. A.15. 17 modes and 3 mode-clusters show statistically significant amount of non-additive brain aging. In most cases, delta is increasing with age (e.g., as can be seen visually in Fig. A.15C for mode $4_{62}$, but some modes are decreasing (e.g., as seen in Fig. A.15D for mode $11_{62}$ ). 


\subsection{Brain-age modes' structural and functional interpretation}

The raw ICA IDP-weights-vectors are plotted in Fig. A.10, with IDPs running along the $x$ axis. FreeSurfer-derived structural IDPs are to the right, functional connectivity (from resting-state fMRI) estimates in the central portion (this is largely - but not completely - empty), and other structural, diffusion MRI and task fMRI measures in the left-most block. These are the raw weights, and we do not discuss this visualisation in greater detail here, because the more compact summary of IDP weights in Fig. 2 is more interpretable, and also the full lists of strongest weights are provided in spreadsheets (see Section 5 ).

Fig. 2 arranges IDPs into logical groupings of distinct types of measures ("modality types" - for the full list of IDPs and their modality groups, see Section 5). For each modality group $j$ comprising $N_{j}$ IDPs, the $N_{\text {modes }} \times N_{I D P s j}$ matrix is fed into ICA to reduce the number of IDPs to a more visually-compact form of IDP "clusters" - thus each column in the figure represents a group of IDPs with similar behaviour across modes. For each IDP modality group, the number of displayed components is data-dependent, utilising the PCA eigenspectrum to determine ICA dimensionality and then retaining ICA components with sufficiently strong maximum weight, though always displaying at least one strongest component (see code linked in Section 5 for full implementational details).

We show separate visualisations for the 62 modes and also the 6 mode-clusters, with the same IDP groupings for each (but separate ICA decompositions, as we did not want either decomposition to influence the other). We can see many clear correspondences between the modes and mode-clusters in compatible ways to those described above. For example, modes $5_{62}$ and $10_{62}$ and mode-cluster $3_{6}$ relate closely to each other, and all are driven by T1 contrast across the grey-white boundary. These figures are discussed in greater detail in Results.

Finally, voxelwise mapping of deltas were estimated to help interpret some modes and relevant imaging modalities. In some cases it was found to be useful to simply correlate delta against the $N_{\text {subjects }} \times N_{\text {voxels }}$ full imaging data, and in other cases we averaged the images from the 1,000 subjects having the lowest (e.g., most negative) delta values, and separately averaged the 1,000 subjects with the largest values, to generate two average images for direct visual comparison. Where appropriate, the imaging data was deconfounded (across-subjects) using the same confound regressors as described above.

\subsection{Associations of brain-age delta with non-imaging measures}

We utilised 8,787 non-imaging, non-genetic measures (which we refer to here as nIDPs - non-imaging-derived phenotypes) from UK Biobank, spanning 16 groups of variable types, including early life factors (such as being breastfed as a baby), lifestyle factors (e.g., exercise, food, alcohol and tobacco variables), physical body measures (e.g., body size, fat, bone density variables and blood assays), cognitive test scores, and health (including mental health) variables (see Figs. A.13, A.14 and online spreadsheets described in Section 5). These variables were automatically curated using the freely avaiable FUNPACK (the FMRIB UKBiobank Normalisation, Parsing And Cleaning Kit https://git.fmrib.ox.ac.uk/fsl/funpack) software; this sorts variables into hand-curated groups, ensures that quantitative variable codings are parsed into at least monotonicallysensible values, and separates categorical variables into multiple binary indicator variables.

The nIDPs were then passed through similar preprocessing as above for IDPs; they were quantile normalised and had all confounds regressed out (including age-related confounds). The one difference here was that, to avoid statistical instability when working with variables that only exist for one sex (e.g., related to menopause), the confound variables were sex-separated before being applied.

The UK Biobank nIDPs have varying amounts of missing data. Here we used 8,787 variables having data from 40 subjects or more. Therefore, the full set of associations of nIDPs against brain-age delta have widely-varying degrees-of-freedom, and taking into account correlation P-values is important (and not just correlation $r$ values). The histogram of non-missing data proportions is shown in Fig. A.11.

To identify the strongest associations between brain-age delta (for each mode and mode-cluster), we used simple Pearson correlation (as described above, both IDPs and nIDPs have been quantile-normalised, i.e., Gaussianised). For each mode/modecluster, we computed correlations between nIDPS and the delta estimates, and also partialled delta estimates (to identify associations between nIDPs and the unique variance in the deltas). We also computed the same sets of associations for just females and just males. In detailed spreadsheets (see Section 5) we report all associations where any of the tests (i.e., using all subjects, and just females, and just males) have a significance value of $-\log _{10} P>5$, although these should be interpreted in the light of the fact that conservative Bonferroni correction across 62 modes and all nIDPs would have a $-\log _{10} P$ threshold of 7.0 , while across 6 mode-clusters this would be 6.0 . 
bioRxiv preprint doi: https://doi.org/10.1101/802686; this version posted January 18,2020 . The copyright holder for this preprint (which was not certified by peer review) is the author/funder, who has granted bioRxiv a license to display the preprint in perpetuity. It is made available under aCC-BY 4.0 International license.

Summary plots simplifying the mapping of modes onto nIDP variables and variable groups (using variable-group-specific ICA) were created in the same manner as described above for IDPs, and form part of Figs. A.13 and A.14

\subsection{GWAS of brain-age delta}

We carried out genome-wide association studies (univariate regressions) of all delta estimates, following the approach used in Elliott et al., 2018. We used the second UK Biobank release of imputed genetic data, comprising over 90 million structural variants (which are primarily SNPs, and are referred to here in general as SNPs for brevity).

We used a minor allele frequency (MAF) threshold of $1 \%$, imputation information score threshold 0.3 and Hardy-Weinberg equilibrium P-value threshold $10^{-7}$. We reduced the subjects used for GWAS to a maximal subset of unrelated subjects with recent British ancestry (to avoid the confounding effects of gross population structure and complex cross-subject covariance). Relatedness was determined by thresholding the kinship matrix at 0.175 , and recent British ancestry was determined using the variable in.white. British.ancestry in the provided genetic data files. 40 population principal components (as supplied by UK Biobank) were used as GWAS confound regressors (again, to avoid the confounding effects of gross population structure).

This QC filtering resulted in a total of 9,812,242 SNPs and 15,952 subjects (samples), which we partitioned at random into a 10,612 subject discovery sample and a 5,340 subject replication sample. GWAS was carried out using BGENIE v1.2 (https://jmarchini.org/bgenie/).

The standard single-phenotype GWAS threshold is $-\log _{10} P=7.5$. Our Manhattan plots (of significance vs. SNPs) show this threshold as well as an adjustment of this for the Bonferroni factor of $62+6$ phenotypes, i.e., $-\log _{10} P=9.33$. This is likely conservative due to correlations across phenotypes (modes and mode-clusters).

After performing the GWAS, we used a method described in [Elliott et al., 2018 to identify meaningfully distinct lead (peak) SNPs, taking into account correlation amongst neighbouring SNPs (linkage disequilibrium). In effect, this identifies distinct clusters of significantly-associated SNPs. This method works by forming a set containing all of the significant SNPs, and then iteratively retains only the top-most significant hit among all SNPs in the set while removing others SNPs within $.25 \mathrm{cM}$ (approximately $250 \mathrm{kbp}$ on average) of the reported peak SNP, terminating after all significant SNPs are removed or retained for reporting.

Fig. 3 shows various Manhattan plots for individual delta estimates as well as all-in-one estimates. Individual Manhattan plots for every mode/mode-cluster, both sex-combined and sex-separated, and for delta and partialled delta, were generated (see Section 5). Summary plots simplifying the mapping of modes onto SNPs and chromosomes (using variable-group-specific ICA) were created in the same manner as described above for IDPs and nIDPs, and form part of Figs. A.13 and A.14

Finally, we ran several distinct kinds of additional genetic analyses. Using the Genome Browser https://genome.ucsc.edu we manually identified RSIDs for all indel variants that we found to have peak associations (that is, all peaks for all modes and all mode-clusters). We then used FUMA https://fuma.ctglab.nl Watanabe et al., 2017 to map SNPs/variants to genes. Next, we used FUMA in conjunction with ANNOVAR http://annovar.openbioinformatics. org/en/latest/ [Wang et al., 2010] to identify SNPs in LD with the peak SNPs, and to functionally annotate them. Taking advantage of gene expression and chromatin databases, we identified eQTL and chromatin mappings/interactions for SNPs and genomic loci, again via FUMA. We also carried out PHEWAS, identifying details of other traits from previous (largely non-UK Biobank) studies having associations with our peak SNPs, via the PHEWAS-atlas/FUMA tool. Finally, we used LD score regression with LDSC v1.0.1 Bulik-Sullivan et al., 2015] (applied separately to each mode/mode-cluster) to estimate genetic (SNP) heritability of all modes and mode-clusters, as well as to estimate genetic co-heritability with Alzheimer's disease [Lambert et al., 2013] and Parkinson's disease [Simón-Sánchez et al., 2009] (see online supplemental materials for AD/PD summary statistics information detail and acknowledgements). All LDSC analysis was done with LD scores computed using the 1000 Genomes European (EUR) subjects [The 1000 Genomes Project Consortium, 2015].

\section{Data and code availability, and additional supplementary tables and figures}

All subject-level data (IDPs, nIDPs and genetics) are available upon application to UK Biobank. The UK Biobank data acquisition MRI protocol, and the image processing and IDP generation pipelines are all freely available https://www.fmrib. ox.ac.uk/ukbiobank/ Additional resources relating to group-average image analysis can be found at https://www.fmrib. 
ox.ac.uk/ukbiobank/ This includes population-average templates for all of the different imaging modalities, and lists/images of all rfMRI nodes and edges.

All code developed for the work reported here (Matlab) is freely available from https://www.fmrib.ox.ac.uk/ukbiobank/ BrainAgingModes.

The same website also contains the following additional supplemental materials: Figures with all modes' / mode-clusters' individual GWAS Manhattan plots; GWAS summary statistics files; rfMRI summary brain images showing visually the brain regions ("nodes") and pairs of brain regions ("edges") significantly associated with all modes and mode-clusters; tables/spreadsheets listing all IDPs used, the strongest nIDP associations with all modes/mode-clusters, the strongest IDP weights for all modes/modeclusters, and the peak GWAS associations (tables can be downloaded or viewed online); and additional genetic analyses including functional annotation, gene expression, associated traits from previous GWAS studies, and genetic heritability/co-heritability results.

\section{Acknowledgments}

SS is supported by a Wellcome Trust Strategic Award 098369/Z/12/Z and a Wellcome Trust Collaborative Award $215573 / Z / 19 / Z$. KM is supported by a Wellcome Trust Senior Research Fellowship 202788/Z/16/Z. The Wellcome Centre for Integrative Neuroimaging (WIN FMRIB) is supported by centre funding from the Wellcome Trust (203139/Z/16/Z). GD is supported by an MRC Career Development Fellowship (MR/K006673/1). This research has been conducted in part using the UK Biobank Resource under Application Number 8107. We are grateful to UK Biobank for making the data available, and to all UK Biobank study participants, who generously donated their time to make this resource possible. Analysis was carried out on the clusters at the Oxford Biomedical Research Computing (BMRC) facility and FMRIB (part of the Wellcome Centre for Integrative Neuroimaging). BMRC is a joint development between the Wellcome Centre for Human Genetics and the Big Data Institute, supported by Health Data Research UK and the NIHR Oxford Biomedical Research Centre.

\section{References}

[Alfaro-Almagro et al., 2018] Alfaro-Almagro, F., Jenkinson, M., Bangerter, N., Andersson, J., Griffanti, L., Douaud, G., Sotiropoulos, S., Jbabdi, S., Hernandez-Fernandez, M., Valee, E., Vidaurre, D., Webster, M., McCarthy, P., Rorden, C., Daducci, A., Alexander, D., Zhang, H., Dragonu, I., Matthews, P., Miller, K., and Smith, S. (2018). Image processing and quality control for the first 10,000 brain imaging datasets from UK Biobank. Neurolmage, 166:400-424.

[Bhalala et al., 2018] Bhalala, O. G., Nath, A. P., Inouye, M., Sibley, C. R., Consortium, U. B. E., et al. (2018). Identification of expression quantitative trait loci associated with schizophrenia and affective disorders in normal brain tissue. PLoS Genetics, 14(8):e1007607.

[Brown et al., 2012] Brown, T. T., Kuperman, J. M., Chung, Y., Erhart, M., McCabe, C., Hagler, D. J., Venkatraman, V. K., Akshoomoff, N., Amaral, D. G., Bloss, C. S., Casey, B. J., Chang, L., Ernst, T. M., Frazier, J. A., Gruen, J. R., Kaufmann, W. E., Kenet, T., Kennedy, D. N., Murray, S. S., Sowell, E. R., Jernigan, T. L., and Dale, A. M. (2012). Neuroanatomical assessment of biological maturity. Curr. Biol., 22(18):1693-1698.

[Bulik-Sullivan et al., 2015] Bulik-Sullivan, B. K., Loh, P. R., Finucane, H. K., Ripke, S., Yang, J., Schizophrenia Working Group of the Psychiatric Genomics Consortium, Patterson, N., Daly, M. J., Price, A. L., and Neale, B. M. (2015). LD Score regression distinguishes confounding from polygenicity in genome-wide association studies. Nat. Genet., 47(3):291-295.

[Chesi et al., 2015] Chesi, A., Mitchell, J. A., Kalkwarf, H. J., Bradfield, J. P., Lappe, J. M., McCormack, S. E., Gilsanz, V., Oberfield, S. E., Hakonarson, H., Shepherd, J. A., Kelly, A., Zemel, B. S., and Grant, S. F. (2015). A trans-ethnic genomewide association study identifies gender-specific loci influencing pediatric aBMD and BMC at the distal radius. Hum. Mol. Genet., 24(17):5053-5059.

[Cole and Franke, 2017] Cole, J. H. and Franke, K. (2017). Predicting age using neuroimaging: Innovative brain ageing biomarkers. Trends in Neurosciences, 40:681-690.

[Cole et al., 2017] Cole, J. H., Poudel, R. P., Tsagkrasoulis, D., Caan, M. W., Steves, C., Spector, T. D., and Montana, G. (2017). Predicting brain age with deep learning from raw imaging data results in a reliable and heritable biomarker. Neurolmage, 163:115-124. 

aCC-BY 4.0 International license.

[Cordes et al., 2016] Cordes, C., Baum, T., Dieckmeyer, M., Ruschke, S., Diefenbach, M. N., Hauner, H., Kirschke, J. S., and Karampinos, D. C. (2016). MR-Based Assessment of Bone Marrow Fat in Osteoporosis, Diabetes, and Obesity. Front Endocrinol (Lausanne), 7:74.

[Coultas et al., 2007] Coultas, L., Terzano, S., Thomas, T., Voss, A., Reid, K., Stanley, E. G., Scott, C. L., Bouillet, P., Bartlett, P., Ham, J., Adams, J. M., and Strasser, A. (2007). Hrk/DP5 contributes to the apoptosis of select neuronal populations but is dispensable for haematopoietic cell apoptosis. J. Cell. Sci., 120(Pt 12):2044-2052.

[Cruchaga et al., 2013] Cruchaga, C., Kauwe, J. S., Harari, O., Jin, S. C., Cai, Y., Karch, C. M., Benitez, B. A., Jeng, A. T., Skorupa, T., Carrell, D., Bertelsen, S., Bailey, M., McKean, D., Shulman, J. M., De Jager, P. L., Chibnik, L., Bennett, D. A., Arnold, S. E., Harold, D., Sims, R., Gerrish, A., Williams, J., Van Deerlin, V. M., Lee, V. M., Shaw, L. M., Trojanowski, J. Q., Haines, J. L., Mayeux, R., Pericak-Vance, M. A., Farrer, L. A., Schellenberg, G. D., Peskind, E. R., Galasko, D., Fagan, A. M., Holtzman, D. M., Morris, J. C., GERAD Consortium, Alzheimer's Disease Neuroimaging Initiative (ADNI), Alzheimer Disease Genetic Consortium (ADGC), and Goate, A. M. (2013). GWAS of cerebrospinal fluid tau levels identifies risk variants for Alzheimer's disease. Neuron, 78(2):256-268.

[Deary et al., 2018] Deary, V., Hagenaars, S. P., Harris, S. E., Hill, W. D., Davies, G., Liewald, D. C., Group, C. C. I., Mclntosh, A., Gale, C., Deary, I., et al. (2018). Genetic contributions to self-reported tiredness. Molecular psychiatry, 23(3):609-620.

[Deming et al., 2017] Deming, Y., Li, Z., Kapoor, M., Harari, O., Del-Aguila, J. L., Black, K., Carrell, D., Cai, Y., Fernandez, M. V., Budde, J., Ma, S., Saef, B., Howells, B., Huang, K. L., Bertelsen, S., Fagan, A. M., Holtzman, D. M., Morris, J. C., Kim, S., Saykin, A. J., De Jager, P. L., Albert, M., Moghekar, A., O'Brien, R., Riemenschneider, M., Petersen, R. C., Blennow, K., Zetterberg, H., Minthon, L., Van Deerlin, V. M., Lee, V. M., Shaw, L. M., Trojanowski, J. Q., Schellenberg, G., Haines, J. L., Mayeux, R., Pericak-Vance, M. A., Farrer, L. A., Peskind, E. R., Li, G., Di Narzo, A. F., Kauwe, J. S., Goate, A. M., and Cruchaga, C. (2017). Genome-wide association study identifies four novel loci associated with Alzheimer's endophenotypes and disease modifiers. Acta Neuropathol., 133(5):839-856.

[Douaud et al., 2014] Douaud, G., Groves, A., Tamnes, C., Westlye, L., Duff, E., Engvig, A., Walhovd, K., James, A., Gass, A., Monsch, A., Matthews, P., Fjell, A., Smith, S., and Johansen-Berg, H. (2014). A common brain network links development, aging and vulnerability to disease. PNAS, 111(49):17648-17653.

[Elliott et al., 2018] Elliott, L., Sharp, K., Alfaro-Almagro, F., Shi, S., Miller, K., Douaud, G., Marchini, J., and Smith, S. (2018). Genome-wide association studies of brain imaging phenotypes in UK Biobank. Nature, 562:210-216.

[Franke et al., 2010] Franke, K., Ziegler, G., Klöppel, S., Gaser, C., and ADNI (2010). Estimating the age of healthy subjects from T1-weighted MRI scans using kernel methods: Exploring the influence of various parameters. Neurolmage, 50:883-892.

[Gasparoni et al., 2018] Gasparoni, G., Bultmann, S., Lutsik, P., Kraus, T. F. J., Sordon, S., Vlcek, J., Dietinger, V., Steinmaurer, M., Haider, M., Mulholland, C. B., Arzberger, T., Roeber, S., Riemenschneider, M., Kretzschmar, H. A., Giese, A., Leonhardt, H., and Walter, J. (2018). DNA methylation analysis on purified neurons and glia dissects age and Alzheimer's disease-specific changes in the human cortex. Epigenetics Chromatin, 11(1):41.

[Groves et al., 2012] Groves, A., Smith, S., Fjell, A., Tamnes, C., Walhovd, K., Douaud, G., Woolrich, M., and Westlye, L. (2012). Benefits of multi-modal fusion analysis on a large-scale dataset: Life-span patterns of inter-subject variability in cortical morphometry and white matter microstructure. Neurolmage, 63(1):365-380.

[GTEx-Consortium, 2017] GTEx-Consortium (2017). Genetic effects on gene expression across human tissues. Nature, 550:204-213.

[Höglinger et al., 2011] Höglinger, G. U., Melhem, N. M., Dickson, D. W., others, and Schellenberg, G. D. (2011). Identification of common variants influencing risk of the tauopathy progressive supranuclear palsy. Nature genetics, 43(7):699.

[Hübel et al., 2019] Hübel, C., Gaspar, H. A., Coleman, J. R., Finucane, H., Purves, K. L., Hanscombe, K. B., Prokopenko, I., investigators, M., Graff, M., Ngwa, J. S., et al. (2019). Genomics of body fat percentage may contribute to sex bias in anorexia nervosa. American Journal of Medical Genetics Part B: Neuropsychiatric Genetics, 180(6):428-438.

[Hyvärinen, 1999] Hyvärinen, A. (1999). Fast and robust fixed-point algorithms for independent component analysis. IEEE Transactions on Neural Networks, 10(3):626-634.

[Ka et al., 2014] Ka, M., Jung, E.-M., Mueller, U., and Kim, W.-Y. (2014). MACF1 regulates the migration of pyramidal neurons via microtubule dynamics and GSK-3 signaling. Developmental biology, 395(1):4-18. 
[Ka et al., 2017] Ka, M., Moffat, J. J., and Kim, W.-Y. (2017). MACF1 controls migration and positioning of cortical GABAergic interneurons in mice. Cerebral cortex, 27(12):5525-5538.

[Kaufmann et al., 2019] Kaufmann, T., van der Meer, D., Doan, N. T., others, and Orhan, F. (2019). Common brain disorders are associated with heritable patterns of apparent aging of the brain. Nat. Neurosci., 22(10):1617-1623.

[Kessler et al., 2016] Kessler, D., Angstadt, M., and Sripada, C. (2016). Growth Charting of Brain Connectivity Networks and the Identification of Attention Impairment in Youth. JAMA Psychiatry, 73(5):481-489.

[Kim et al., 2015] Kim, T., Park, J., Jo, S., Kim, M., Nojima, H., and Ahn, Y. (2015). Effects of low-fat diet and aging on metabolic profiles of Creb314 knockout mice. Nutrition \& diabetes, 5(8):e179.

[Lambert et al., 2013] Lambert, J. C., Ibrahim-Verbaas, C. A., Harold, D., Others, and Amouyel, P. (2013). Meta-analysis of 74,046 individuals identifies 11 new susceptibility loci for Alzheimer's disease. Nat. Genet., 45(12):1452-1458.

[Le et al., 2018] Le, T., Kuplicki, R., McKinney, B., Yeh, H.-W., Thompson, W., Paulus, M., and Tulsa1000 (2018). A nonlinear simulation framework supports adjusting for age when analyzing BrainAGE. Front. Aging Neurosci.

[Lewis et al., 2018] Lewis, J. D., Evans, A. C., and Tohka, J. (2018). T1 white/gray contrast as a predictor of chronological age, and an index of cognitive performance. Neuroimage, 173:341-350.

[Liem et al., 2017] Liem, F., Varoquaux, G., Kynast, J., Beyer, F., Kharabian Masouleh, S., Huntenburg, J. M., Lampe, L., Rahim, M., Abraham, A., Craddock, R. C., Riedel-Heller, S., Luck, T., Loeffler, M., Schroeter, M. L., Witte, A. V., Villringer, A., and Margulies, D. S. (2017). Predicting brain-age from multimodal imaging data captures cognitive impairment. Neuroimage, 148:179-188.

[Liu et al., 2016] Liu, L., Watanabe, N., Akatsu, H., and Nishimura, M. (2016). Neuronal expression of ILEl/FAM3C and its reduction in Alzheimer's disease. Neuroscience, 330:236-246.

[Loskutova et al., 2009] Loskutova, N., Honea, R. A., Vidoni, E. D., Brooks, W. M., and Burns, J. M. (2009). Bone density and brain atrophy in early Alzheimer's disease. J. Alzheimers Dis., 18(4):777-785.

[Maekawa et al., 2015] Maekawa, M., Iwayama, Y., Ohnishi, T., Toyoshima, M., Shimamoto, C., Hisano, Y., Toyota, T., Balan, S., Matsuzaki, H., Iwata, Y., et al. (2015). Investigation of the fatty acid transporter-encoding genes SLC27A3 and SLC27A4 in autism. Scientific reports, 5:16239.

[Miller et al., 2016] Miller, K., Alfaro-Almagro, F., Bangerter, N., Thomas, D., Yacoub, E., Xu, J., Bartsch, A., Jbabdi, S., Sotiropoulos, S., Andersson, J., Griffanti, L., Douaud, G., Okell, T., Weale, P., Dragonu, I., Garratt, S., Hudson, S., Collins, R., Jenkinson, M., Matthews, P., and Smith, S. (2016). Multimodal population brain imaging in the UK Biobank prospective epidemiological study. Nature Neuroscience, 19:1523-1536.

[Moers et al., 2008] Moers, A., Nurnberg, A., Goebbels, S., Wettschureck, N., and Offermanns, S. (2008). Galpha12/Galpha13 deficiency causes localized overmigration of neurons in the developing cerebral and cerebellar cortices. Mol. Cell. Biol., 28(5):1480-1488.

[Mollink et al., 2019] Mollink, J., Smith, S., Elliott, L., Kleinnijenhuis, M., Hiemstra, M., Alfaro-Almagro, F., Marchini, J., van Cappellen van Walsum, A.-M., Jbabdi, S., and Miller, K. (2019). The spatial correspondence and genetic influence of interhemispheric connectivity with white matter microstructure. Nature Neuroscience, 22:809-19.

[Moverare-Skrtic et al., 2015] Moverare-Skrtic, S., Wu, J., Henning, P., Gustafsson, K. L., Sjogren, K., Windahl, S. H., Koskela, A., Tuukkanen, J., Borjesson, A. E., Lagerquist, M. K., Lerner, U. H., Zhang, F. P., Gustafsson, J. A., Poutanen, M., and Ohlsson, C. (2015). The bone-sparing effects of estrogen and WNT16 are independent of each other. Proc. Natl. Acad. Sci. U.S.A., 112(48):14972-14977.

[Ning et al., 2018] Ning, K., Zhao, L., Matloff, W., Sun, F., and Toga, A. W. (2018). Association of brain age with smoking, alcohol consumption, and genetic variants. bioRxiv, page 469924.

[O'Flaherty, 2000] O'Flaherty, E. J. (2000). Modeling normal aging bone loss, with consideration of bone loss in osteoporosis. Toxicol. Sci., 55(1):171-188.

[Richard et al., 2018] Richard, G., Kolskår, K., Sanders, A.-M., Kaufmann, T., Petersen, A., Doan, N. T., Monereo Sánchez, J., Alnæs, D., Ulrichsen, K. M., Dørum, E. S., Andreassen, O. A., Nordvik, J. E., and Westlye, L. T. (2018). Assessing distinct patterns of cognitive aging using tissue-specific brain age prediction based on diffusion tensor imaging and brain morphometry. PeerJ, 6:e5908. 
[Ruddy et al., 2019] Ruddy, R. M., Adams, K. V., and Morshead, C. M. (2019). Age-and sex-dependent effects of metformin on neural precursor cells and cognitive recovery in a model of neonatal stroke. Science advances, 5(9):eaax1912.

[Salat et al., 2009] Salat, D. H., Lee, S. Y., Van der Kouwe, A., Greve, D. N., Fischl, B., and Rosas, H. D. (2009). Ageassociated alterations in cortical gray and white matter signal intensity and gray to white matter contrast. Neuroimage, 48(1):21-28.

[Simón-Sánchez et al., 2009] Simón-Sánchez, J., Schulte, C., Bras, J. M., others, and Gasser, T. (2009). Genome-wide association study reveals genetic risk underlying Parkinson's disease. Nat. Genet., 41(12):1308-1312.

[Smith et al., 2019] Smith, S., Vidaurre, D., Alfaro-Almagro, F., Nichols, T., and Miller, K. (2019). Estimation of brain age delta from brain imaging. Neurolmage, 200:528-539.

[The 1000 Genomes Project Consortium, 2015] The 1000 Genomes Project Consortium (2015). A global reference for human genetic variation. Nature, 526(7571):68-74.

[Vidal-Piñeiro et al., 2016] Vidal-Piñeiro, D., Walhovd, K. B., Storsve, A. B., Grydeland, H., Rohani, D. A., and Fjell, A. M. (2016). Accelerated longitudinal gray/white matter contrast decline in aging in lightly myelinated cortical regions. Human brain mapping, 37(10):3669-3684.

[Vinke et al., 2018] Vinke, E. J., de Groot, M., Venkatraghavan, V., Klein, S., Niessen, W. J., Ikram, M. A., and Vernooij, M. W. (2018). Trajectories of imaging markers in brain aging: the Rotterdam Study. Neurobiol. Aging, 71:32-40.

[Wang et al., 2010] Wang, K., Li, M., and Hakonarson, H. (2010). ANNOVAR: functional annotation of genetic variants from high-throughput sequencing data. Nucleic Acids Res., 38(16):e164.

[Wang et al., 2018] Wang, R., Tang, Z., Sun, X., Wu, L., Wang, J., Zhong, Y., and Xiao, Z. (2018). White Matter Abnormalities and Correlation With Severity in Normal Tension Glaucoma: A Whole Brain Atlas-Based Diffusion Tensor Study. Invest. Ophthalmol. Vis. Sci., 59(3):1313-1322.

[Watanabe et al., 2017] Watanabe, K., Taskesen, E., van Bochoven, A., and Posthuma, D. (2017). Functional mapping and annotation of genetic associations with FUMA. Nat Commun, 8(1):1826. 


\section{A Supplementary figures}
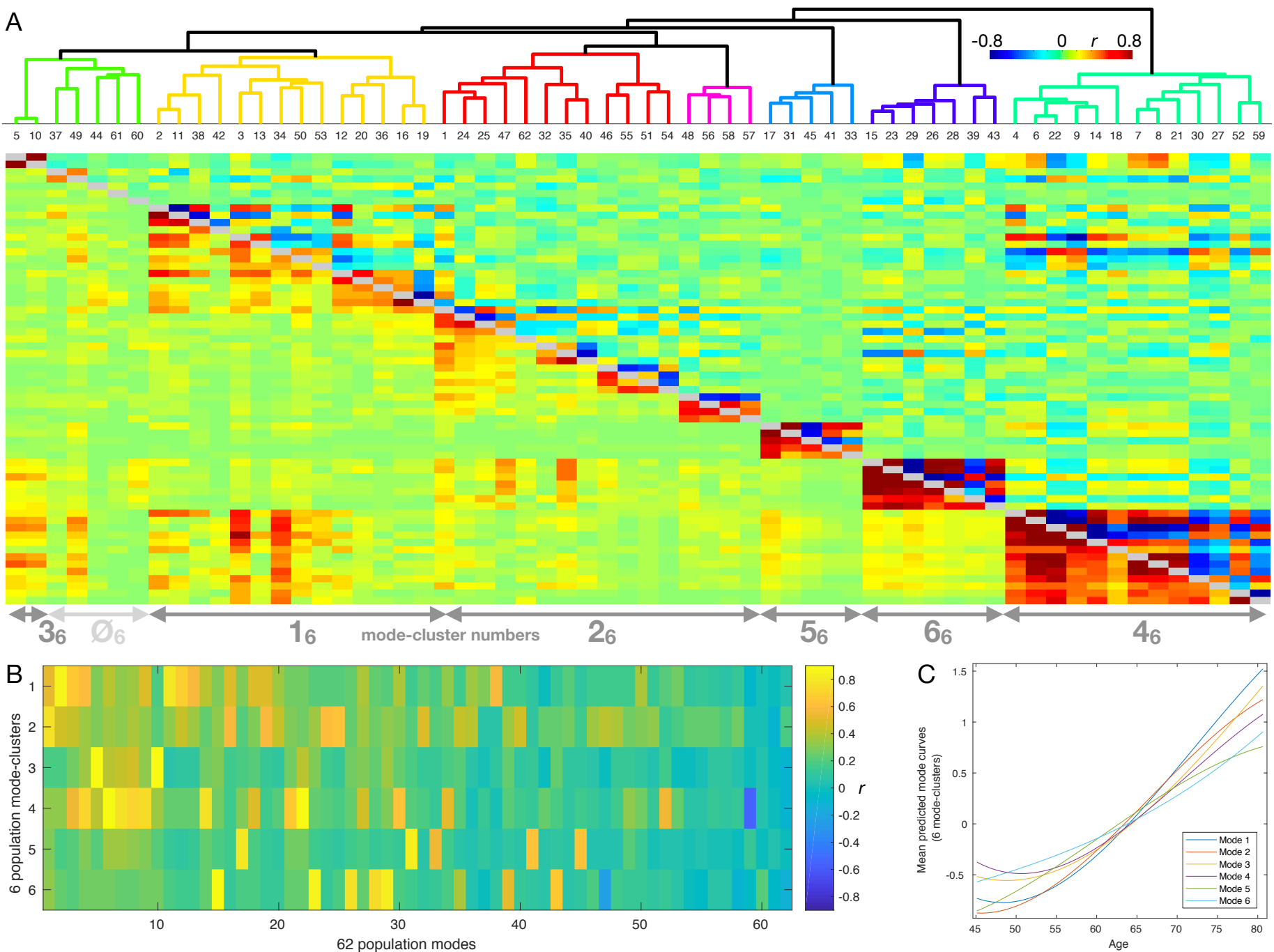

Figure A.1: Hierarchical clustering of the 62 brain-aging modes, and their mapping onto 6 lower-dimensional modeclusters. A) Hierarchical clustering carried out on the basis of the absolute values of the correlations (of subject-weights) between modes (shown below the diagonal). The same correlation values, but with negatives shown in blue, are shown above the diagonal. Arrows show approximate correspondence between hierarchical clusters and low-dimensional mode-clusters (with mode $1_{62}$ appearing to a reasonable extent in mode-clusters $1_{6}$ and $2_{6}$ ). B) The precise mapping between 62 modes and 6 mode-clusters, quantified by correlating subject-weight-vectors between the two. C) The 6 mode-cluster mean aging curves.

\section{B Supplementary comments on body size and other "baseline" causal factors in IDPs and brain aging}

We now include a simple discussion of the opposing signs of involvement of the various body-size-related variables seen for mode-clusters $1_{6}$ and 2 .

The typical starting point for modelling brain aging (e.g., see [Smith et al., 2019]) is

$$
Y_{B}=Y+\delta=f(X)=X \beta,
$$

where actual age is $Y$ (an $N_{\text {subjects }} \times 1$ vector), brain age is $Y_{B}$ and the brain-age delta is $\delta=Y_{B}-Y$. The imaging data 
bioRxiv preprint doi: https://doi.org/10.1101/802686; this version posted January 18,2020 . The copyright holder for this preprint (which was not certified by peer review) is the author/funder, who has granted bioRxiv a license to display the preprint in perpetuity. It is made available under aCC-BY 4.0 International license.
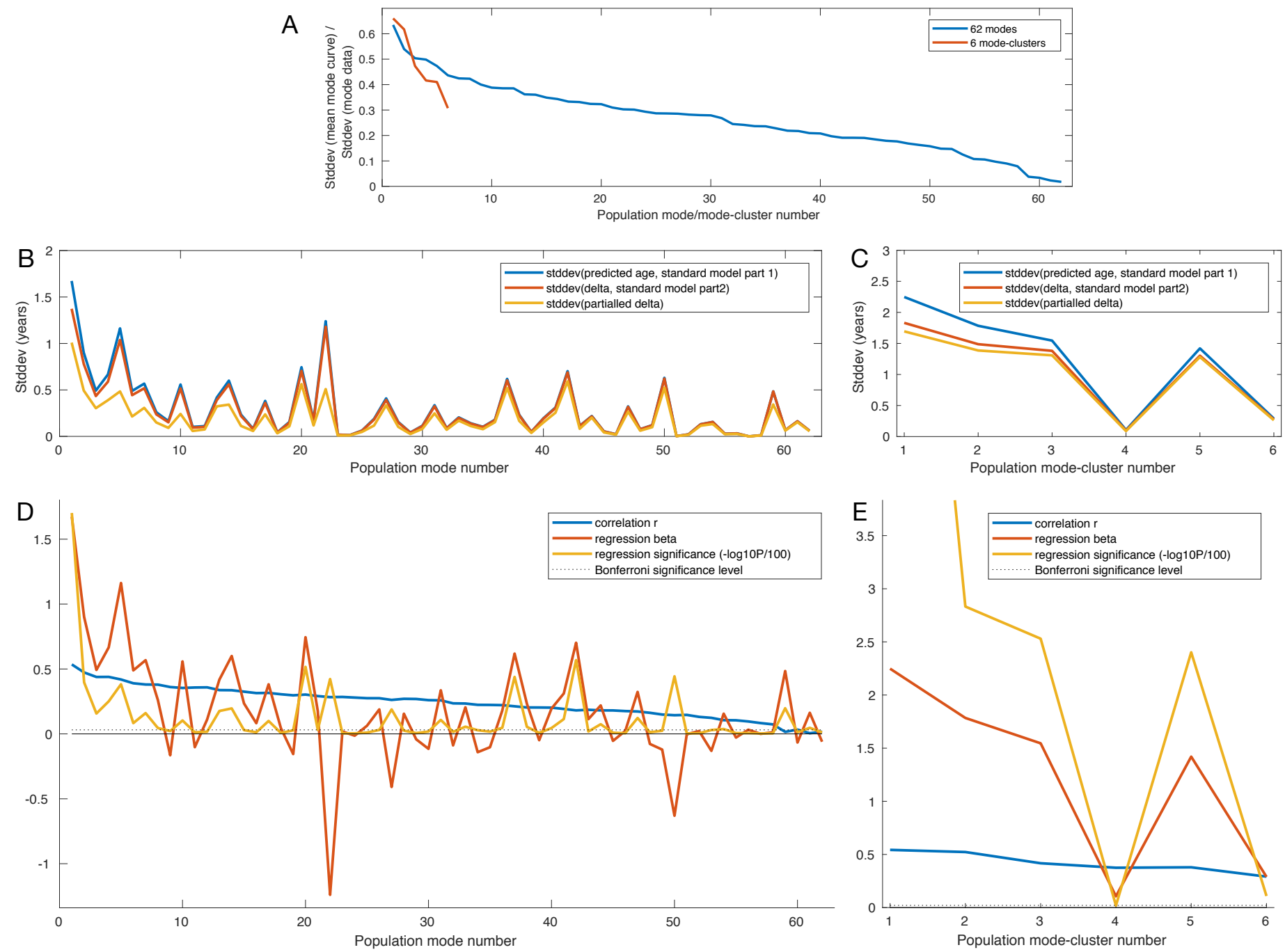

Figure A.2: Model standard deviations, age correlations and age regressions for all modes and mode-clusters. See Methods for details. Units for the y axes in D,E are not marked, as they are different for the different curves, and made clear for each curve in the figure legends.

matrix is $X$, which has $N_{\text {subjects }}$ rows and $D$ columns; the columns are features from the imaging data, and might be different voxels, or different IDPs (imaging-derived phenotypes - summary measures of brain structure and function), or different modes.

Here we treat $X$ as a single feature, for example, total volume of grey matter. We might expect grey matter volume $G$ for subject $i$ to depend both on overall body size as well as age-related atrophy, and hence follow a form like:

$$
G_{i}=b B_{i}-Y_{i}\left(\alpha_{\text {average }}+\alpha_{i}\right)
$$

where $B_{i}$ is a subject's "baseline" body size, $b$ the coefficient relating body size to grey matter volume, $\alpha_{\text {average }}$ is the population average rate of atrophy (the reciprocal of $\beta$ in general), and $\alpha_{i}$ is the subject's deviation (in atrophy rate) from the population average. By definition here $b$ and $\alpha_{\text {average }}$ are positive.

Now, in such cases where the imaging feature is negatively correlated with age (hence the minus sign above), the mode preprocessing used in our modelling flips the sign of the mode so that the subject weights are positively correlated with age (see Methods). Hence we have:

$$
\begin{array}{r}
X_{i}=-G_{i}=-b B_{i}+Y_{i}\left(\alpha_{\text {average }}+\alpha_{i}\right) \\
X_{i} \beta=-b \beta B_{i}+Y_{i}+Y_{i} \alpha_{i} \beta \\
\delta_{i}=X_{i} \beta-Y_{i}=\Delta_{i}-b_{2} B_{i}
\end{array}
$$


bioRxiv preprint doi: https://doi.org/10.1101/802686; this version posted January 18,2020 . The copyright holder for this preprint (which was not certified by peer review) is the author/funder, who has granted bioRxiv a license to display the preprint in perpetuity. It is made available under aCC-BY 4.0 International license.

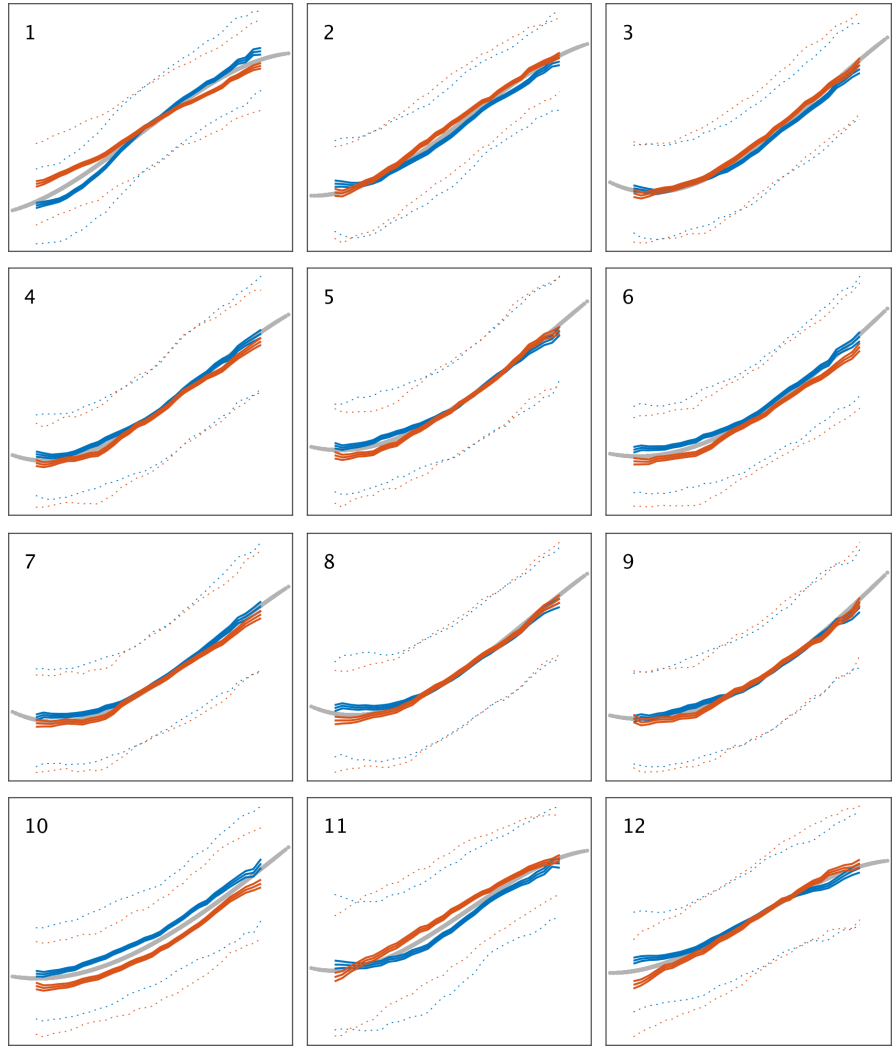

Sex-separated age curves for modes 1-12
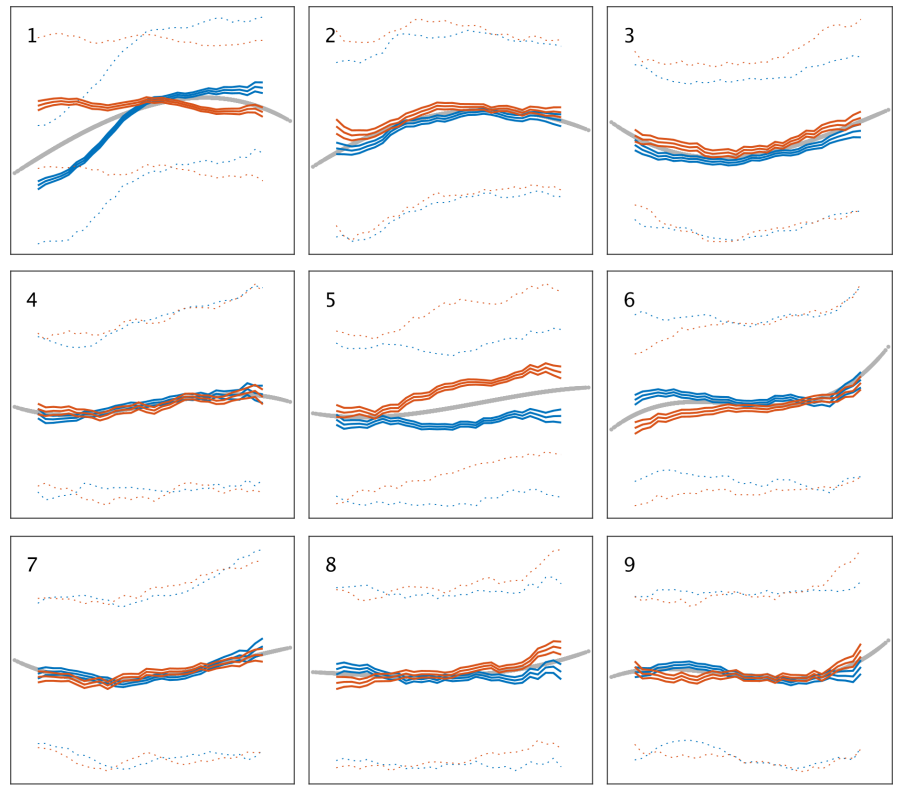

9
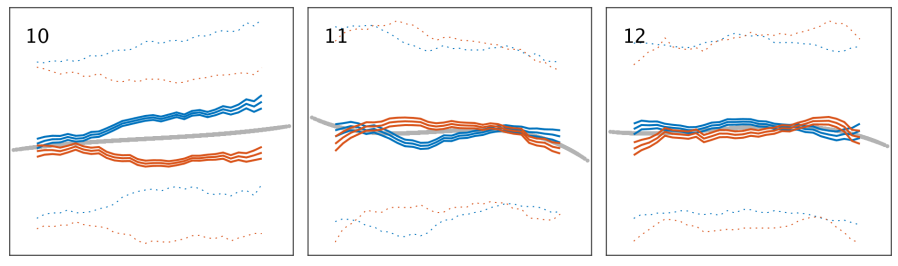

Sex-separated age curves for partialled modes 1-12

Figure A.3: Sex-separated mean age curves for modes 1-12. Left: Mean curves for modes 1-12, from the original subjectweight-vector mode values. The solid grey curve is the fitted age curve using the cubic age model (all subjects combined, see Methods). The blue curves show the females-only sliding-window mean age curve (see Methods); the central line is the mean, and the two outer solid lines show the standard error for the mean. The dotted lines show the 25th and 75th percentiles of the data. Orange curves show the same quantities for males. For clarity, axes are not annotated; in all cases, the $x$-axis is age (from 45-81y), and the y-axis is the unitless subject-weight-vector values. Right: The same plots are shown for the partialled subject-weight-vectors (the original subject-weights after regressing out all other modes).

where $b_{2}=b \beta$ (i.e., is typically a positive coefficient, although multiple-regression age prediction from multiple modes can result in negative $\beta$, as discussed above) and $\Delta_{i}=Y_{i} \alpha_{i} \beta$ is the aspect of the brain age delta that is separate from the effect of the baseline body size (i.e., relates to the ongoing atrophy).

Hence estimated $\delta$ does correctly reflect the atrophy-related delta; however, additionally, between-subject variations in baseline body size result in a larger body giving an apparently lower $\delta$. In cases where the IDP/mode changes are positively correlated with aging (e.g., CSF volume, as in mode-cluster $1_{6}$ ), there is no negative sign above, and no sign-flipping for the mode, and hence the apparent effect of body size is not reversed. Of course, to further complicate matters, some "baseline" or "background" factors (such as socio-economic status) may well have a significant causal role both in baseline IDP/mode values as well as aging rate.

Put more simply and qualitatively, a subject with large body size will have large baseline CSF, and the brain-age modelling will therefore likely consider that large body size is a "bad thing" with respect to mode-cluster $1_{6}$; on the other hand, the same subject will have large baseline grey matter, and the brain-age modelling will therefore consider that large body size is a "good thing" with respect to mode-cluster $2_{6}$. For such cases of course neither simplistic conclusion is appropriate.

Note that in the simpler case where an nIDP is more directly related to an IDP or mode (e.g., as is found with alcohol and smoking), the signs of the associations between $\delta$ and the IDP and the nIDPs are all simply consistent and easily interpretable. For example, for mode-cluster $1_{6}$, CSF volume is positively correlated with $\delta$ (higher CSF volume is indeed a "bad thing"); for mode-cluster $2_{6}$, grey matter volume is negatively correlated with $\delta$ (grey matter volume is a "good thing"), and for both mode-clusters, alcohol and smoking are positively correlated with $\delta$ (they are both "bad things"). 
bioRxiv preprint doi: https://doi.org/10.1101/802686; this version posted January 18,2020 . The copyright holder for this preprint (which was not certified by peer review) is the author/funder, who has granted bioRxiv a license to display the preprint in perpetuity. It is made available under aCC-BY 4.0 International license.
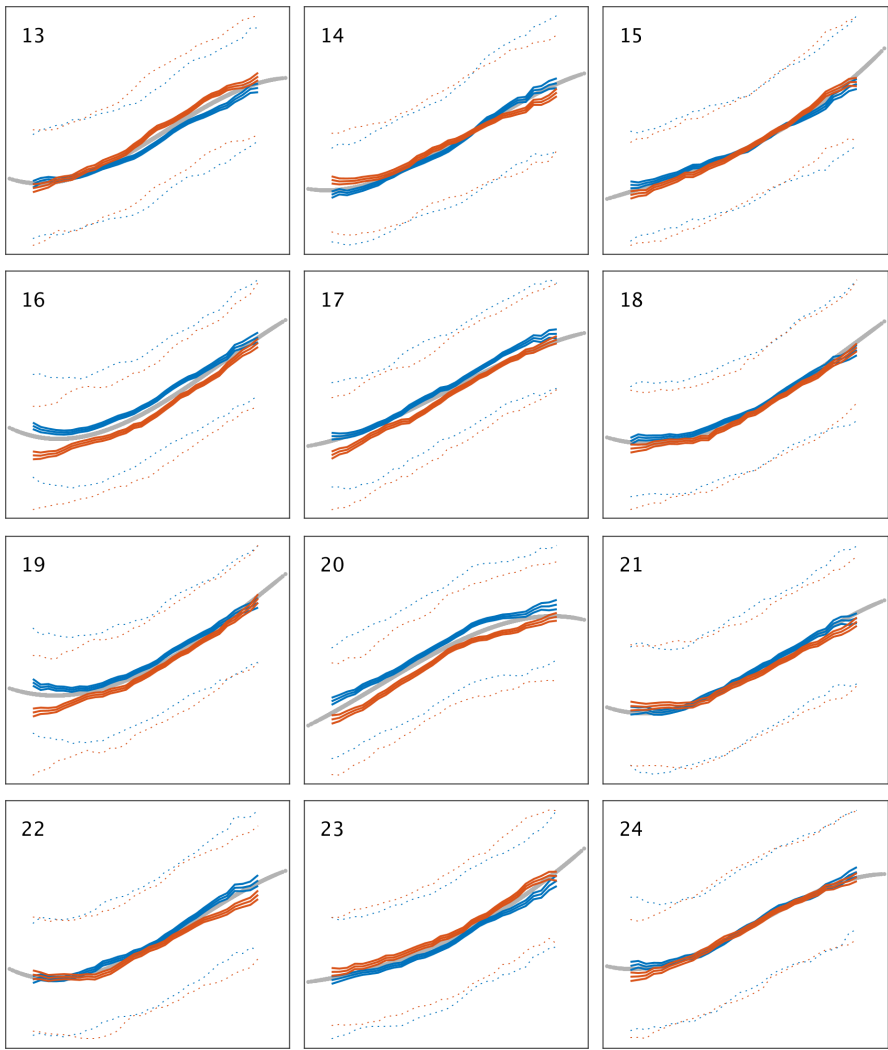

Sex-separated age curves for modes $13-24$
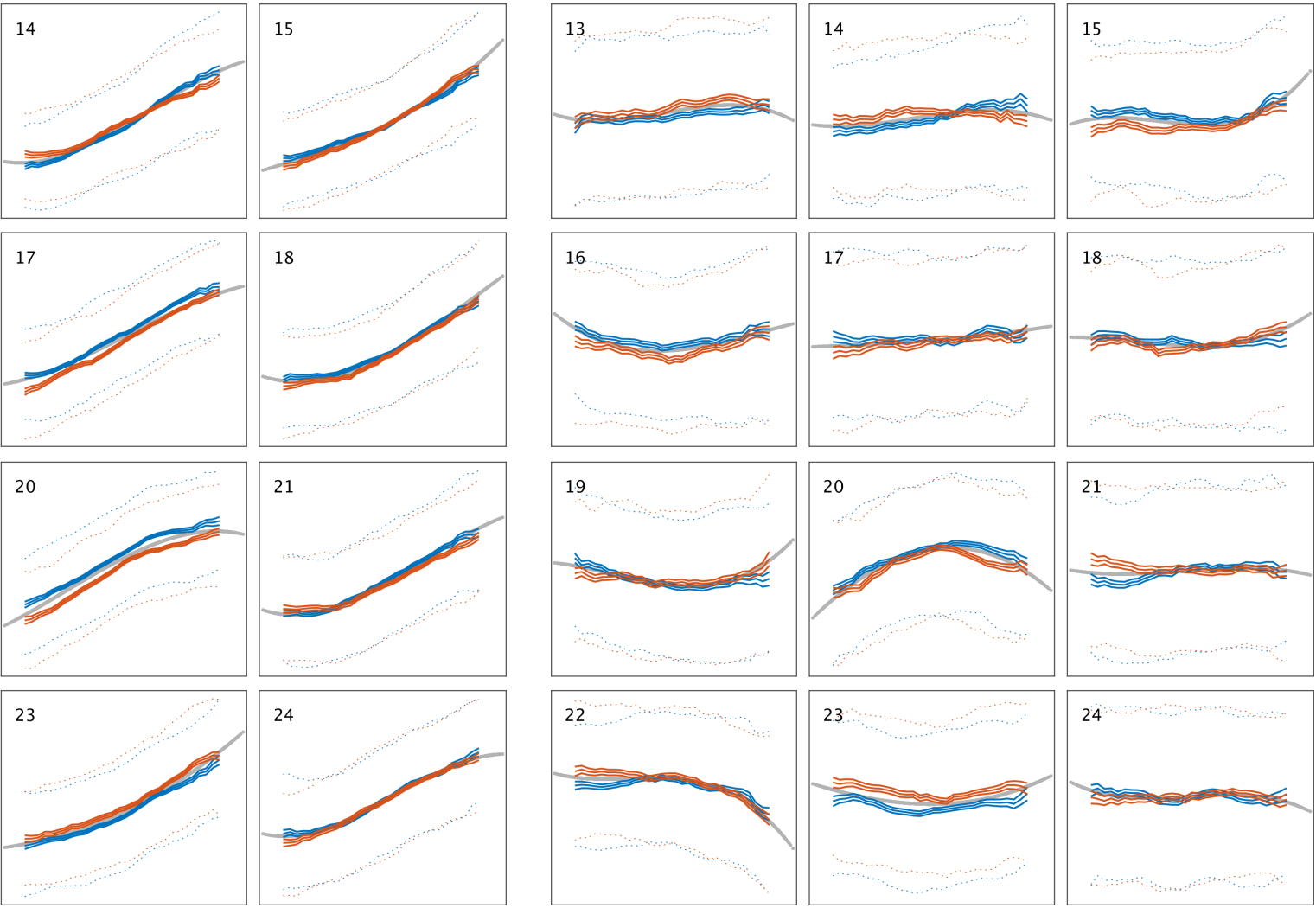

Figure A.4: Sex-separated mean age curves for modes 13-24. See Fig. A.3 for details.
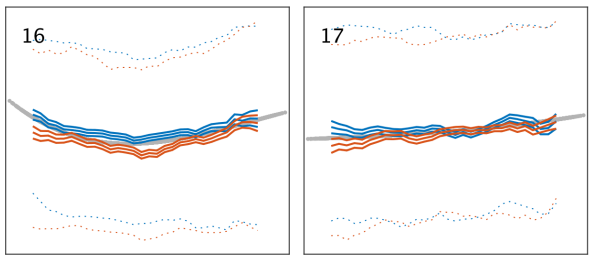

18
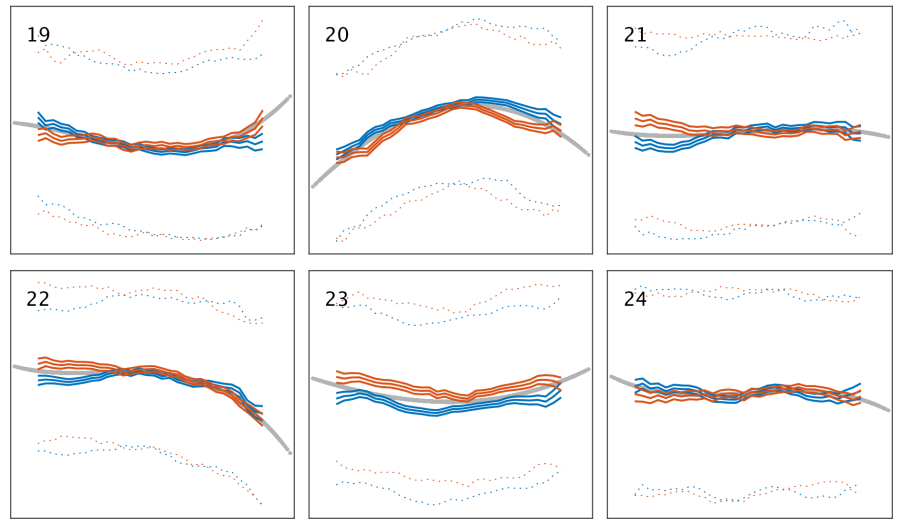

Sex-separated age curves for partialled modes 13-24

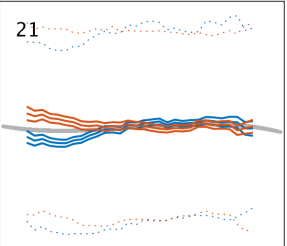

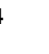


bioRxiv preprint doi: https://doi.org/10.1101/802686; this version posted January 18,2020 . The copyright holder for this preprint (which was not certified by peer review) is the author/funder, who has granted bioRxiv a license to display the preprint in perpetuity. It is made available under aCC-BY 4.0 International license.
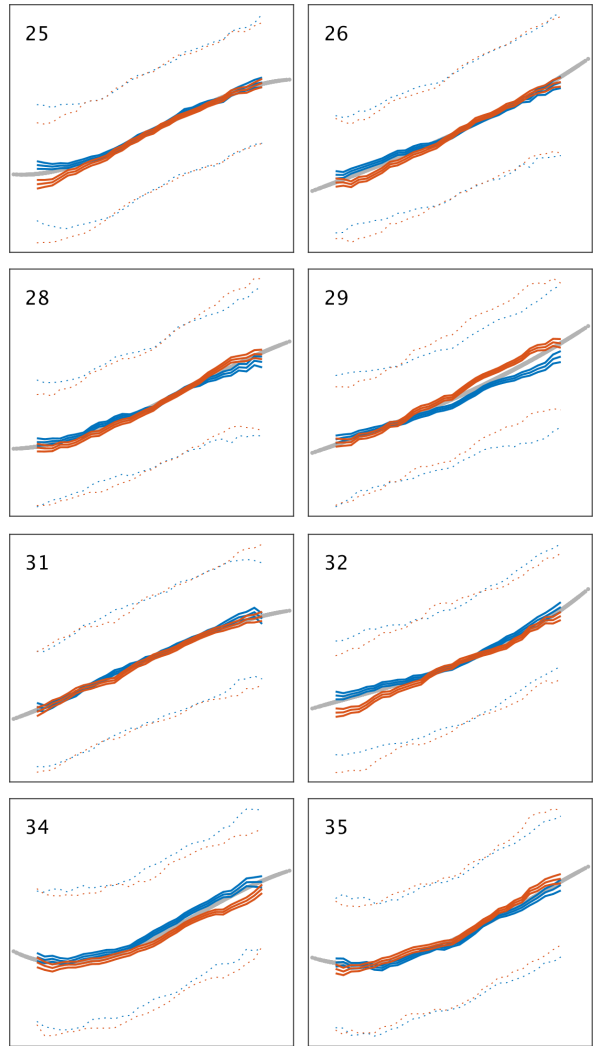

Sex-separated age curves for modes $25-36$
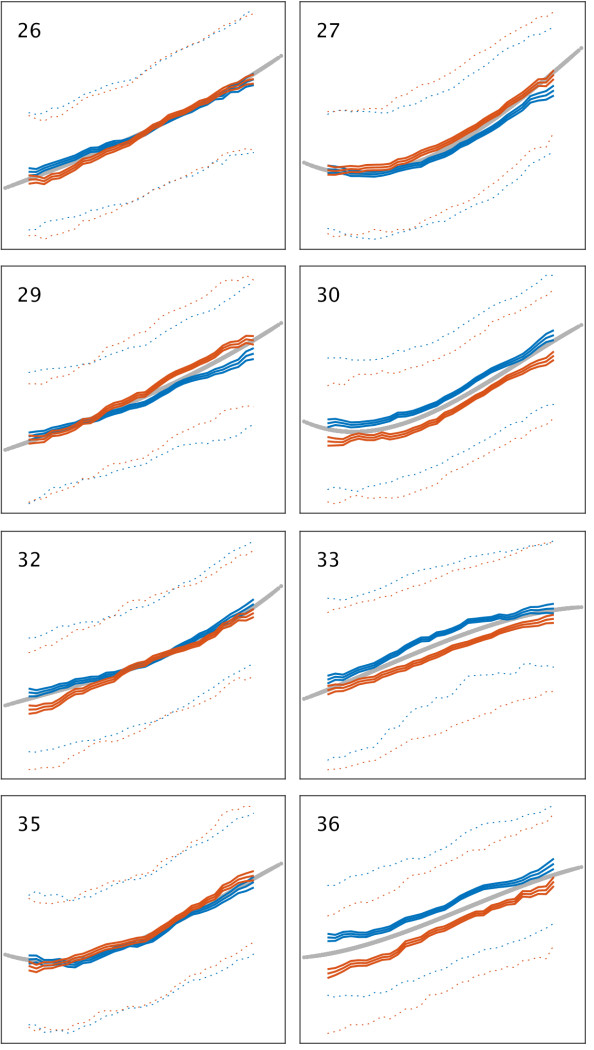

Figure A.5: Sex-separated mean age curves for modes 25-36. See Fig. A.3 for details.
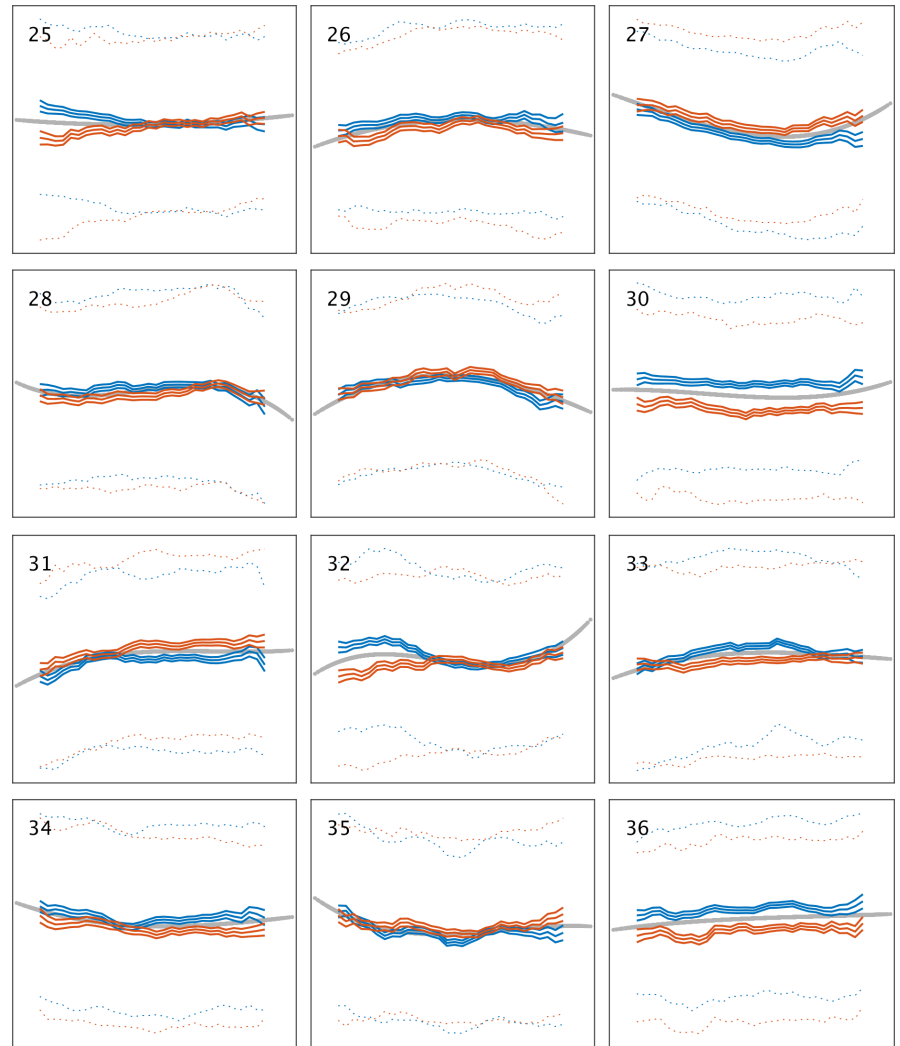

36

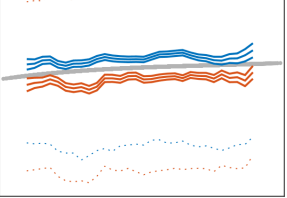

Sex-separated age curves for partialled modes 25-36 
bioRxiv preprint doi: https://doi.org/10.1101/802686; this version posted January 18,2020 . The copyright holder for this preprint (which was not certified by peer review) is the author/funder, who has granted bioRxiv a license to display the preprint in perpetuity. It is made available under aCC-BY 4.0 International license.
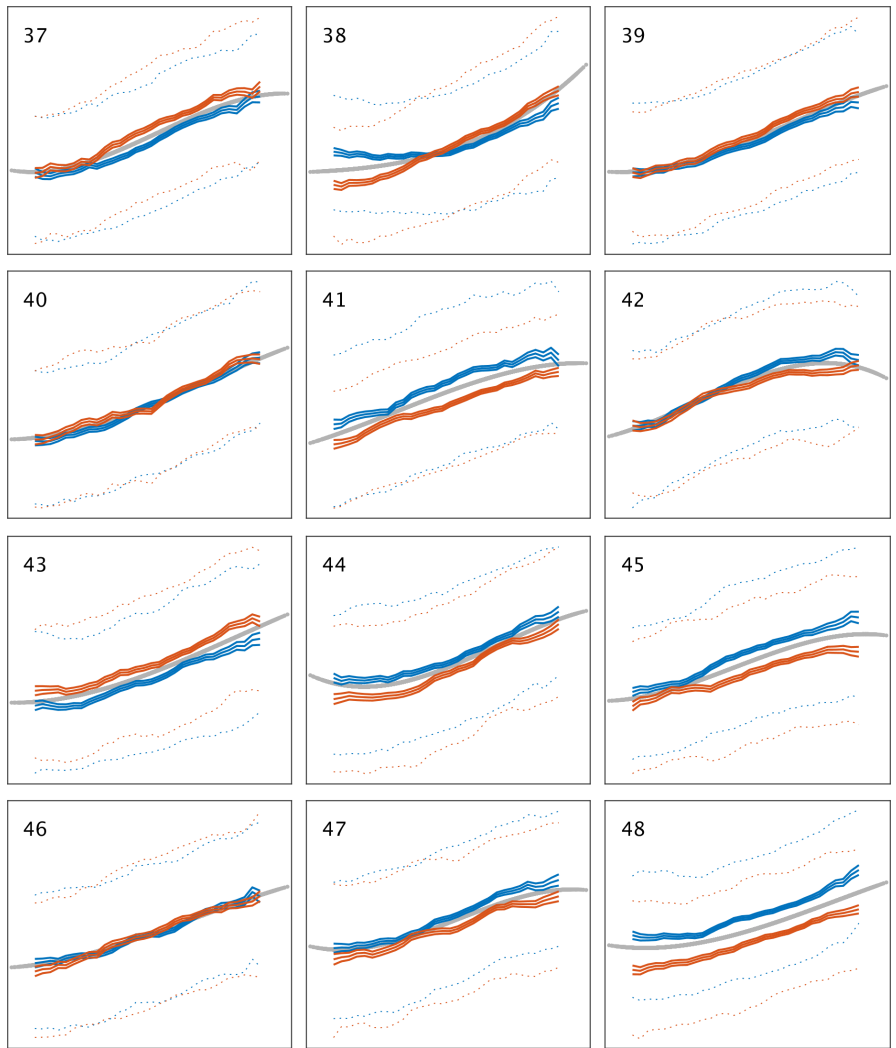

Sex-separated age curves for modes $37-48$
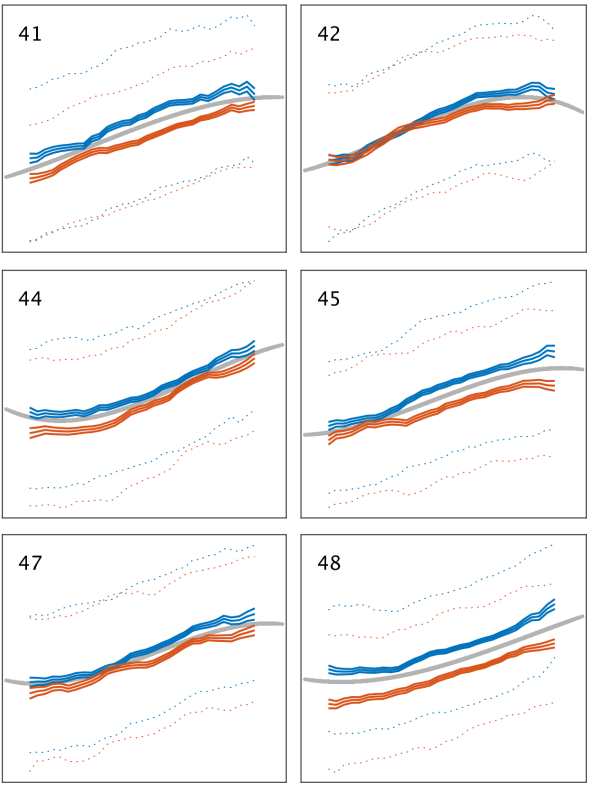

Figure A.6: Sex-separated mean age curves for modes 37-48. See Fig. A.3 for details.
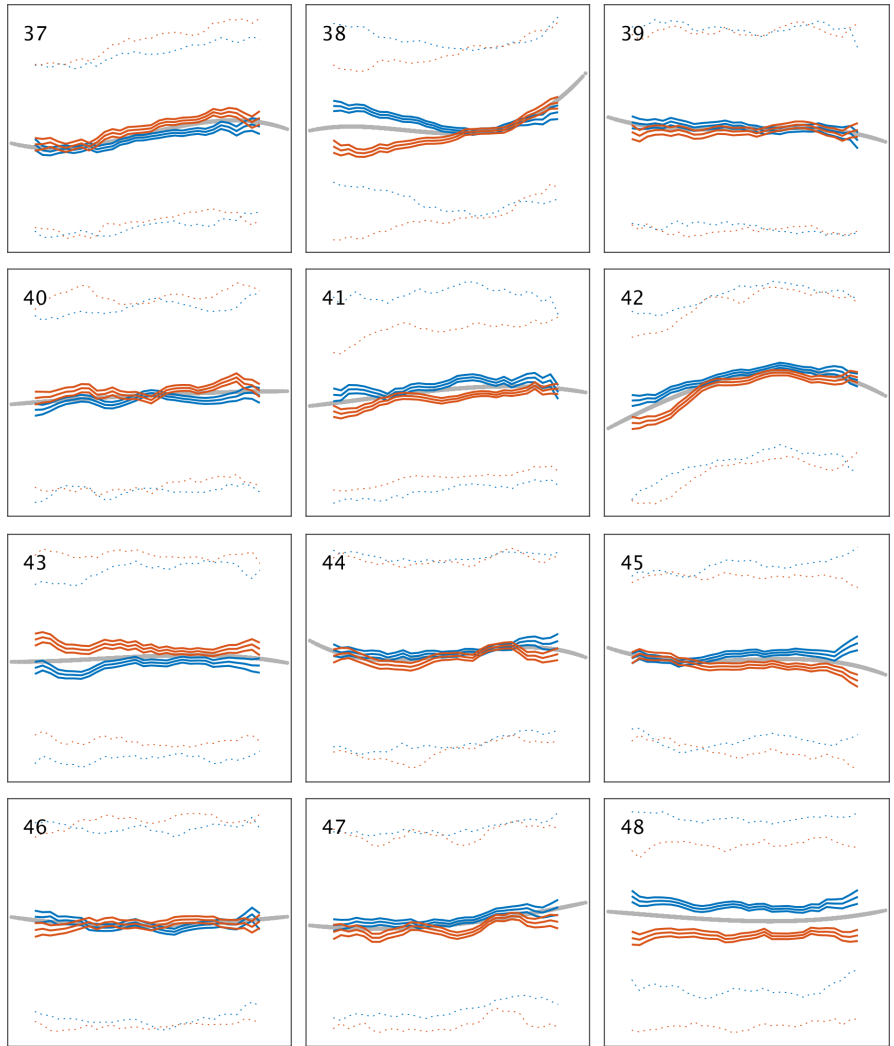

Sex-separated age curves for partialled modes 37-48 
bioRxiv preprint doi: https://doi.org/10.1101/802686; this version posted January 18,2020 . The copyright holder for this preprint (which was not certified by peer review) is the author/funder, who has granted bioRxiv a license to display the preprint in perpetuity. It is made available under aCC-BY 4.0 International license.
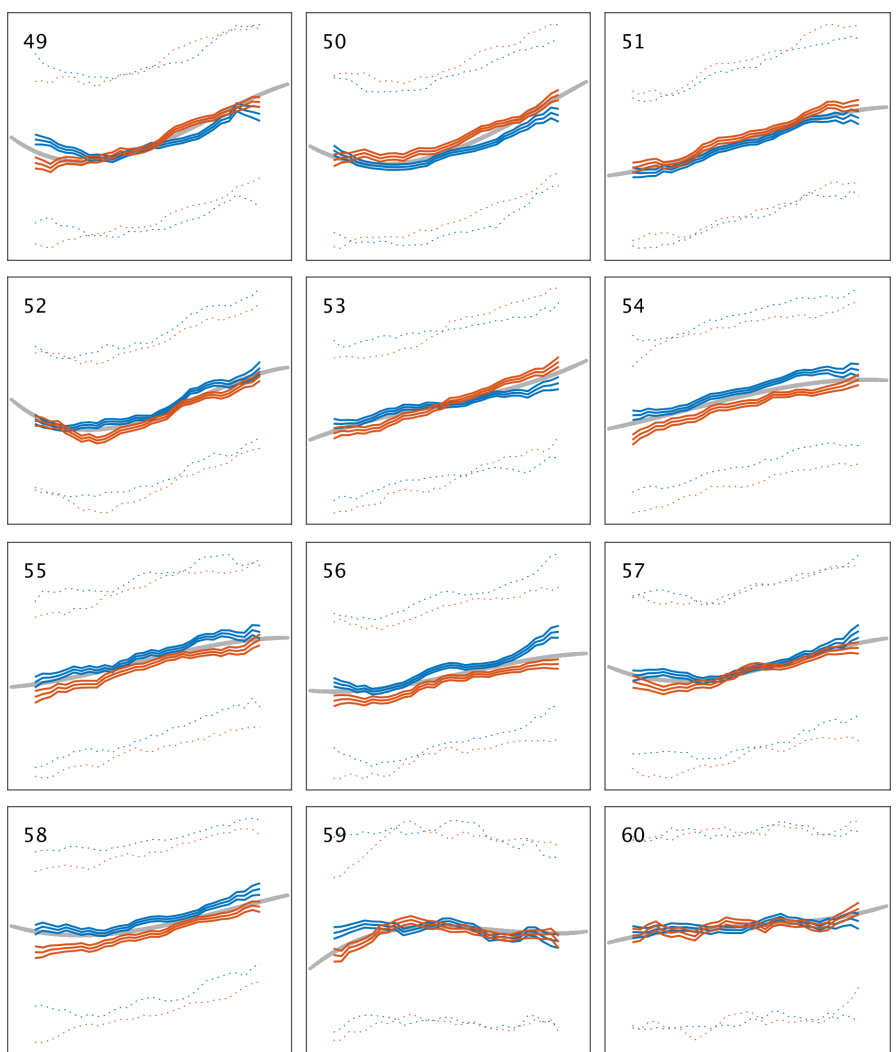

Sex-separated age curves for modes $49-60$
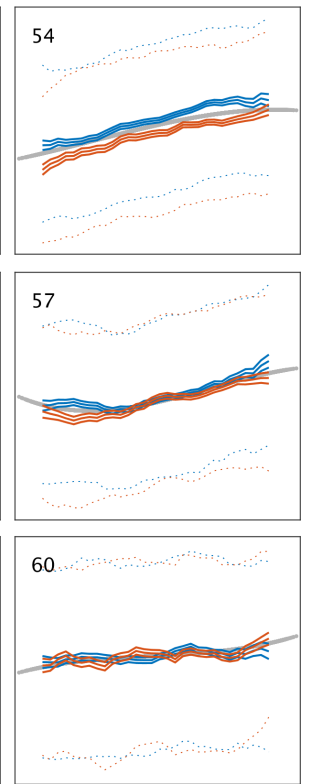
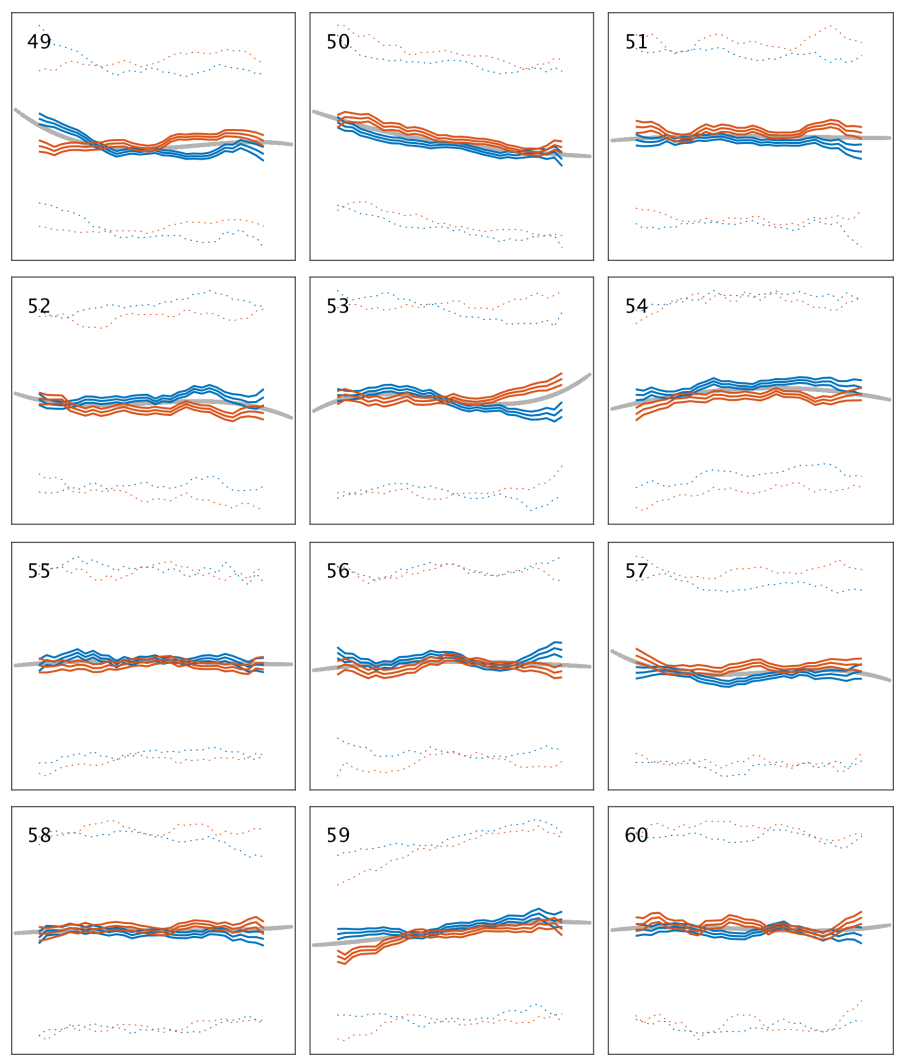

Sex-separated age curves for partialled modes $49-60$

Figure A.7: Sex-separated mean age curves for modes 49-60. See Fig. A.3 for details.

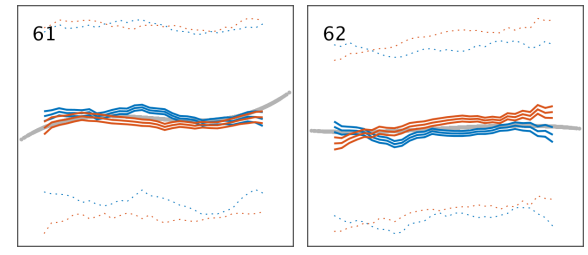

Sex-separated age curves for modes 61-62

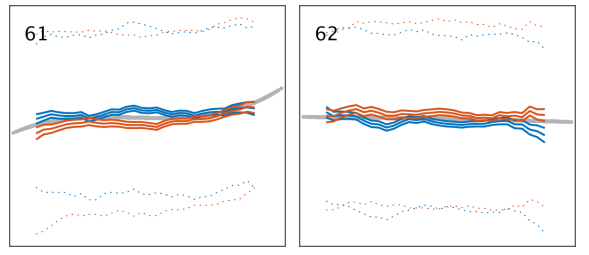

Sex-separated age curves for partialled modes 61-62

Figure A.8: Sex-separated mean age curves for modes 61-62. See Fig. A.3 for details. 
bioRxiv preprint doi: https://doi.org/10.1101/802686; this version posted January 18,2020 . The copyright holder for this preprint (which was not certified by peer review) is the author/funder, who has granted bioRxiv a license to display the preprint in perpetuity. It is made available under aCC-BY 4.0 International license.
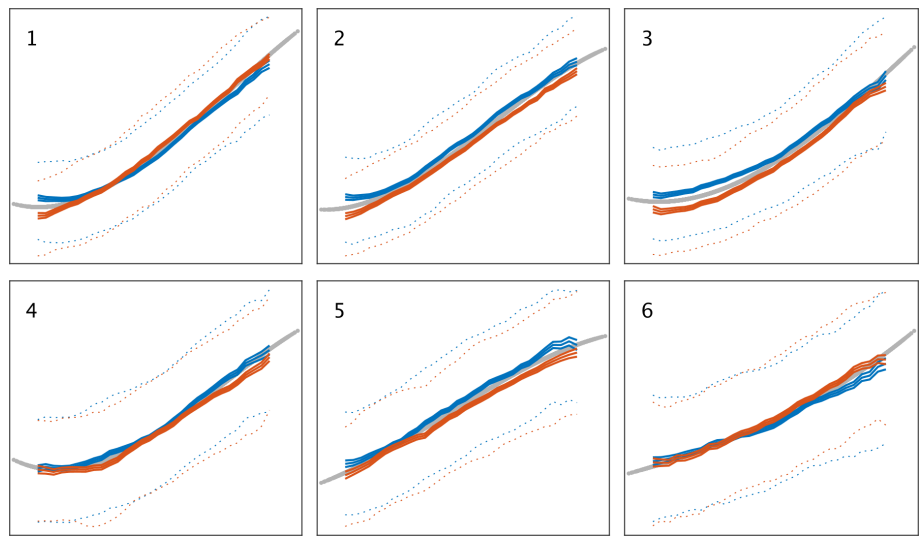

Sex-separated age curves for mode-clusters
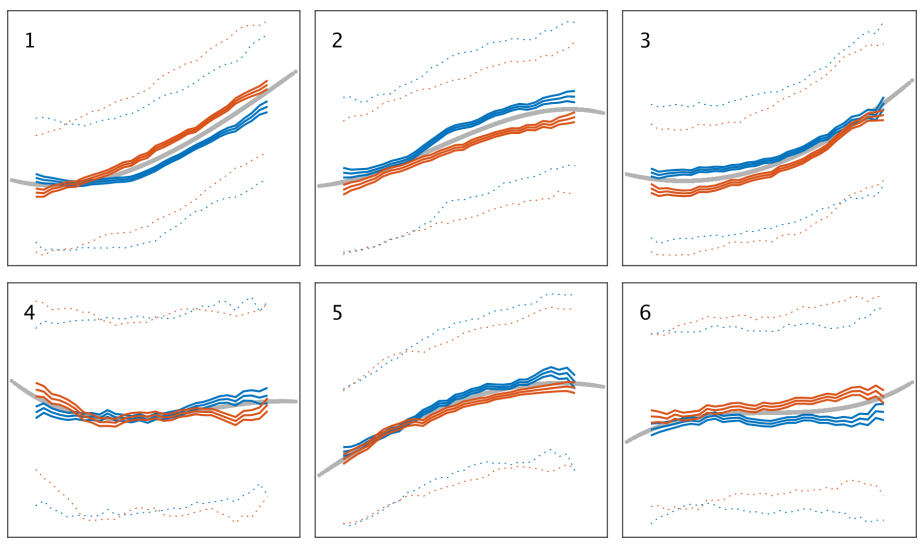

Sex-separated age curves for partialled mode-clusters

Figure A.9: Sex-separated mean age curves for mode-clusters 1-6. See Fig. A.3 for details. 
bioRxiv preprint doi: https://doi.org/10.1101/802686; this version posted January 18, 2020. The copyright holder for this preprint (which was not certified by peer review) is the author/funder, who has granted bioRxiv a license to display the preprint in perpetuity. It is made available under aCC-BY 4.0 International license.
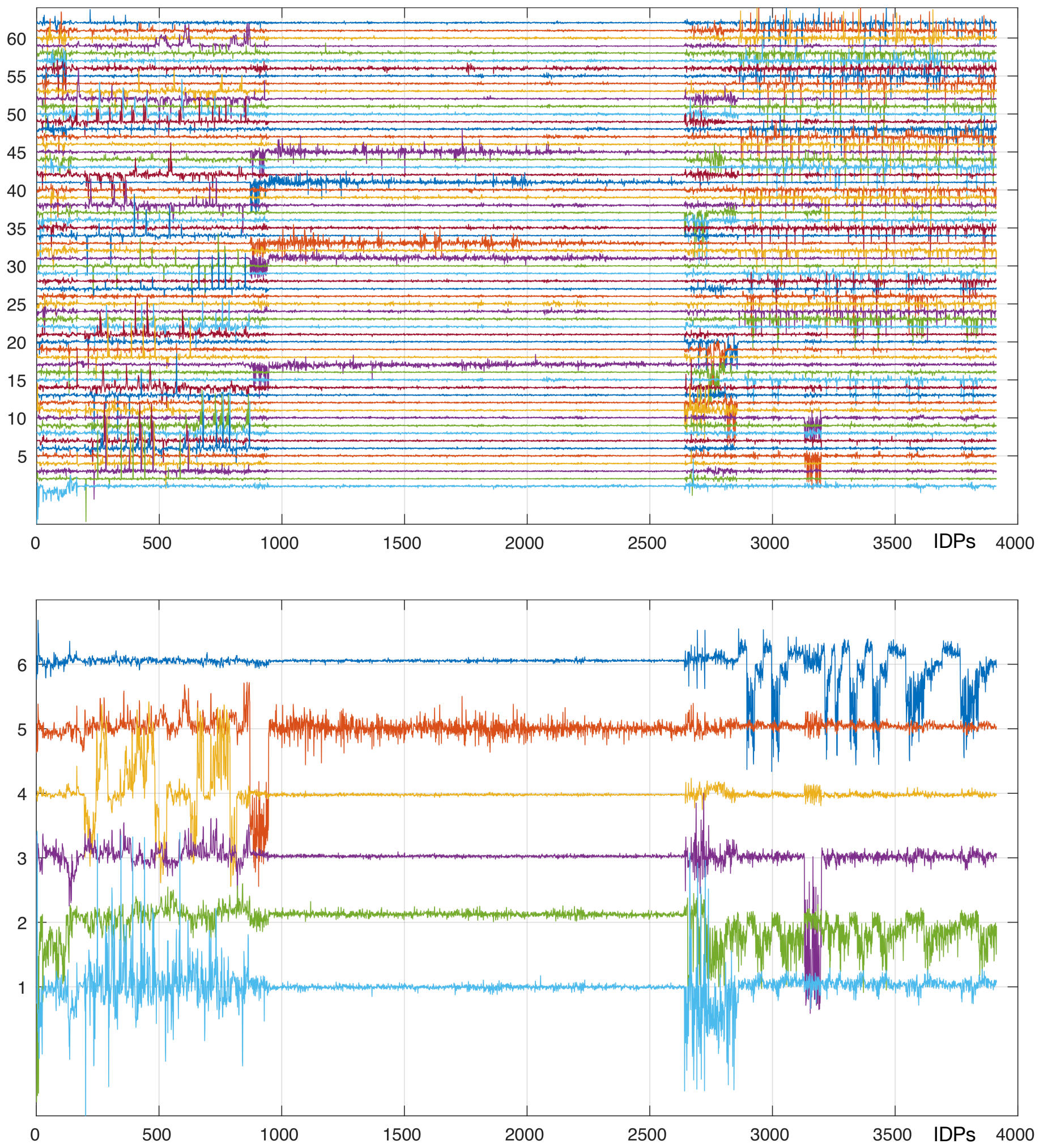

Figure A.10: Mapping of brain-aging modes and mode-clusters onto individual IDPs. See Methods for details, and Section 5 for the complete listing of IDPs (x axis) and tables listing the strongest weights from these mappings. See Fig. 2 for a simpler, more interpretable summary of this, where the $x$ axis is reduced to different classes of IDPs. 
bioRxiv preprint doi: https://doi.org/10.1101/802686; this version posted January 18,2020 . The copyright holder for this preprint (which was not certified by peer review) is the author/funder, who has granted bioRxiv a license to display the preprint in perpetuity. It is made available under aCC-BY 4.0 International license.

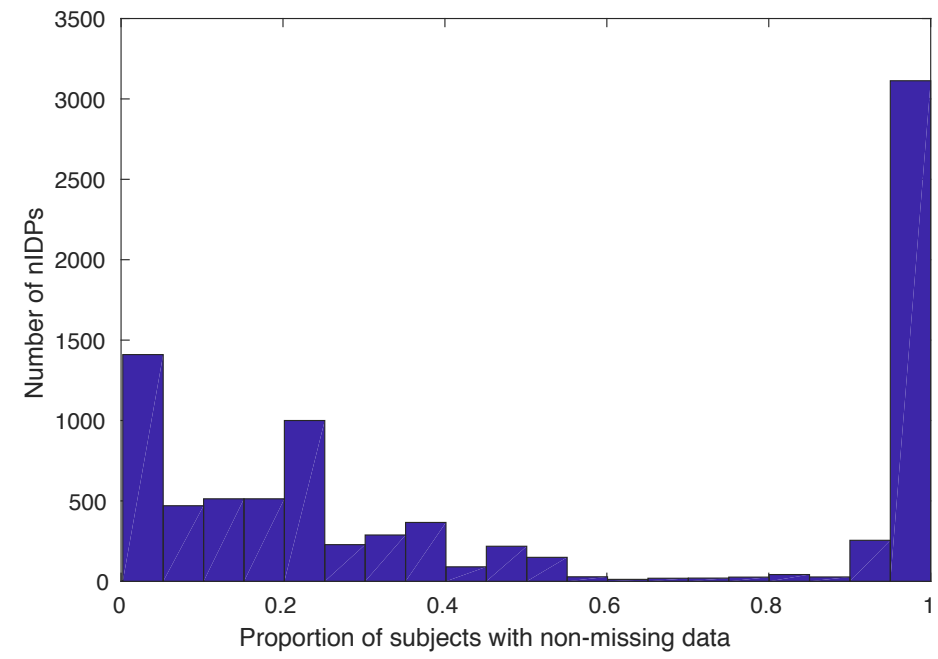

Figure A.11: Histogram of proportions of subjects of (non-missing) data for each nIDP (non-imaging-derived phenotypes). nIDPs are not retained if fewer than 40 subjects have data present. 
bioRxiv preprint doi: https://doi.org/10.1101/802686; this version posted January 18,2020 . The copyright holder for this preprint (which was not certified by peer review) is the author/funder, who has granted bioRxiv a license to display the preprint in perpetuity. It is made available under aCC-BY 4.0 International license.
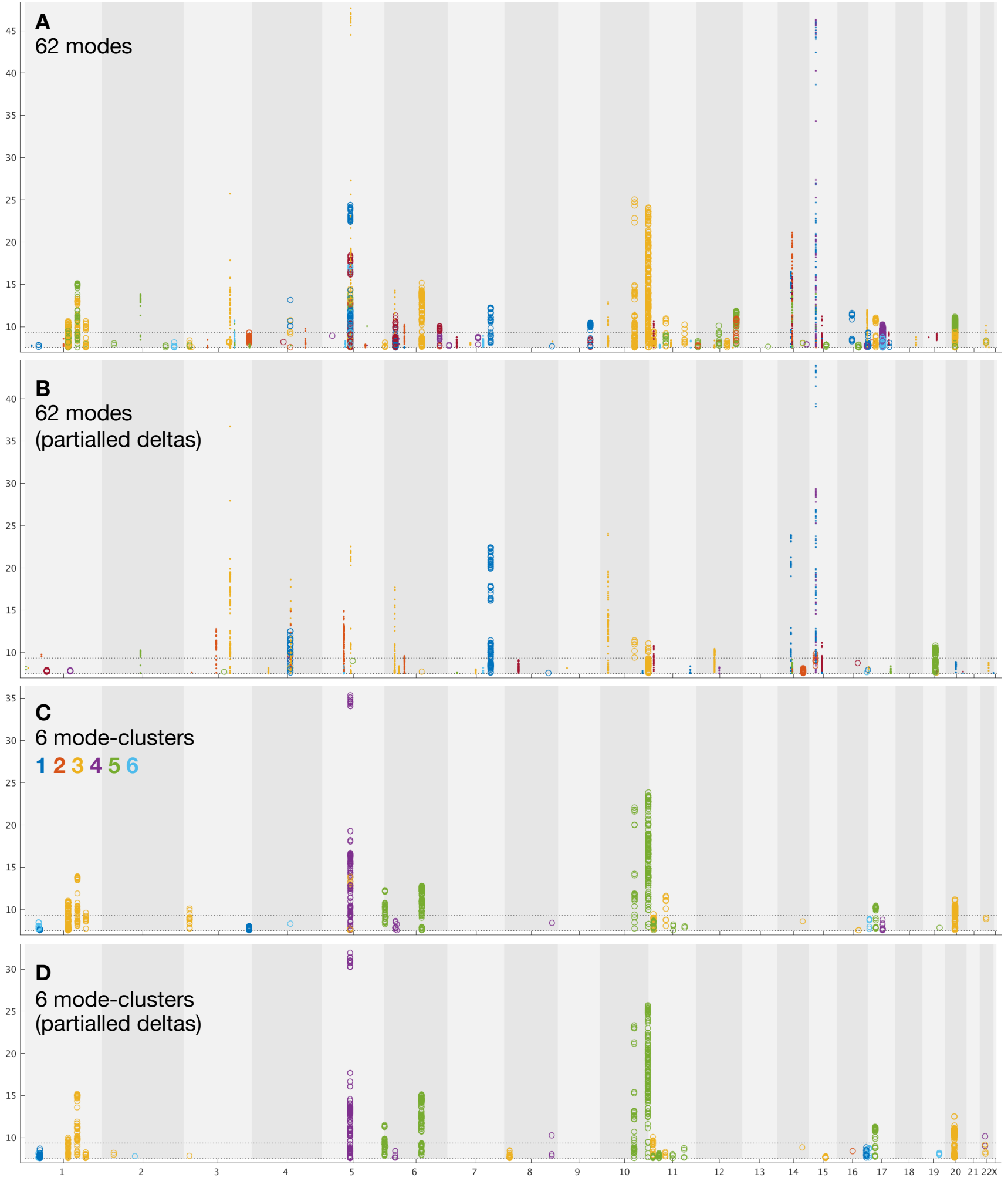

Figure A.12: Summary plots for GWAS of brain aging. See main text Fig. 3 for general plot overview. A) Separate GWAS for deltas from each of the 62 modes of brain aging. This is only different than Fig. $3 \mathrm{~A}$ in that the lower threshold is raised to 7.5 to exclude all non-significant associations, and there is no upper truncation excluding "redundant" higher SNPs. B) GWAS for 62 modes' partialled delta estimates. C) GWAS for deltas from each of the 6 mode-clusters of brain aging. D) GWAS for 6 mode-clusters'partialled delta estimates. In all cases, see links in Section 5 for a complete set of individual modes/mode-cluster GWAS plots. 
bioRxiv preprint doi: https://doi.org/10.1101/802686; this version posted January 18,2020 . The copyright holder for this preprint (which was not certified by peer review) is the author/funder, who has granted bioRxiv a license to display the preprint in perpetuity. It is made available under aCC-BY 4.0 International license.

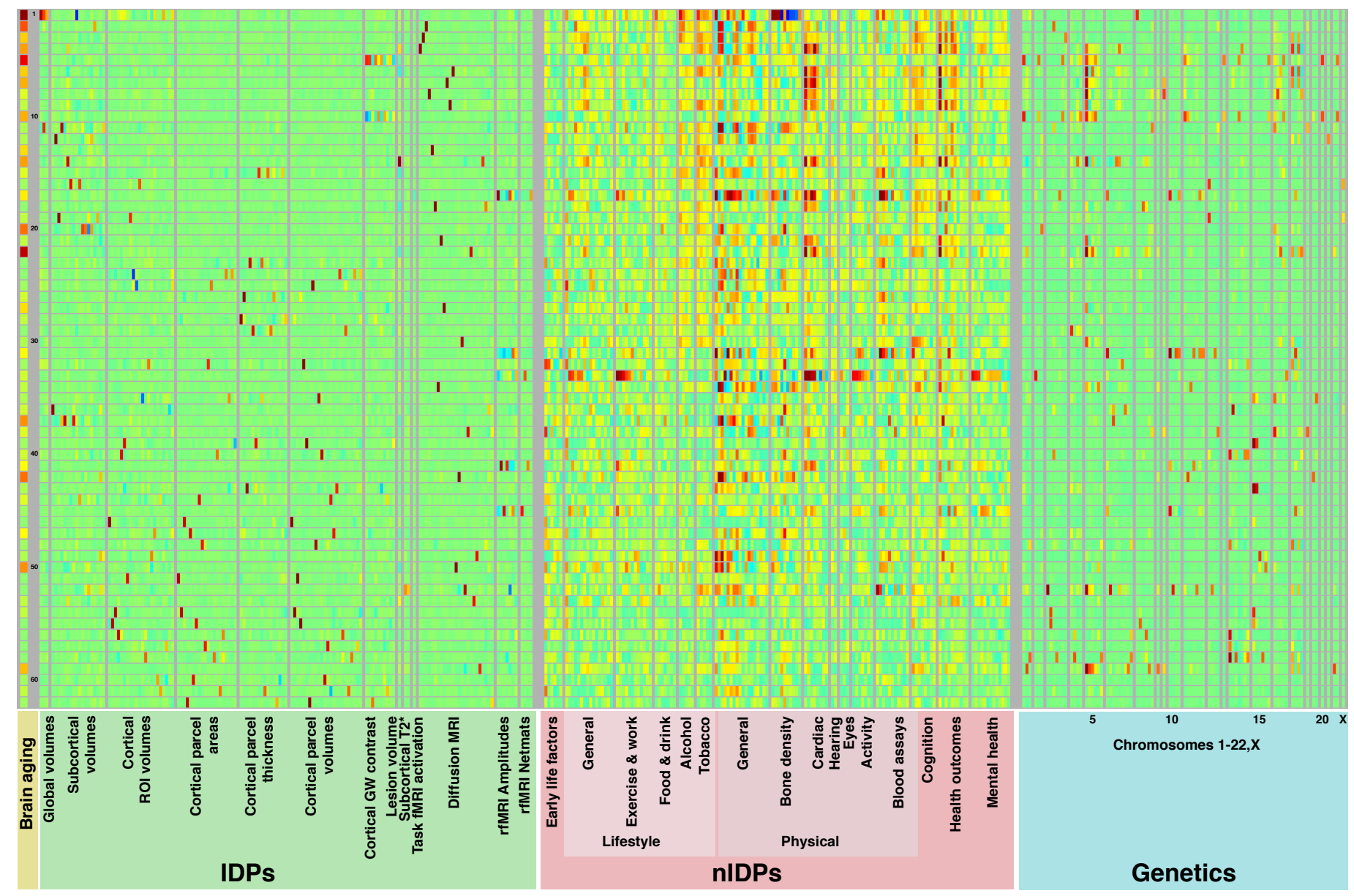

Figure A.13: Mapping of brain-aging modes onto classes of IDPs, nIDPs and chromosomes. This is an expansion of Fig. 2 (upper), to also show how the 62 brain-aging modes map onto non-imaging measures and genetics. 
bioRxiv preprint doi: https://doi.org/10.1101/802686; this version posted January 18,2020 . The copyright holder for this preprint (which was not certified by peer review) is the author/funder, who has granted bioRxiv a license to display the preprint in perpetuity. It is made available under aCC-BY 4.0 International license.
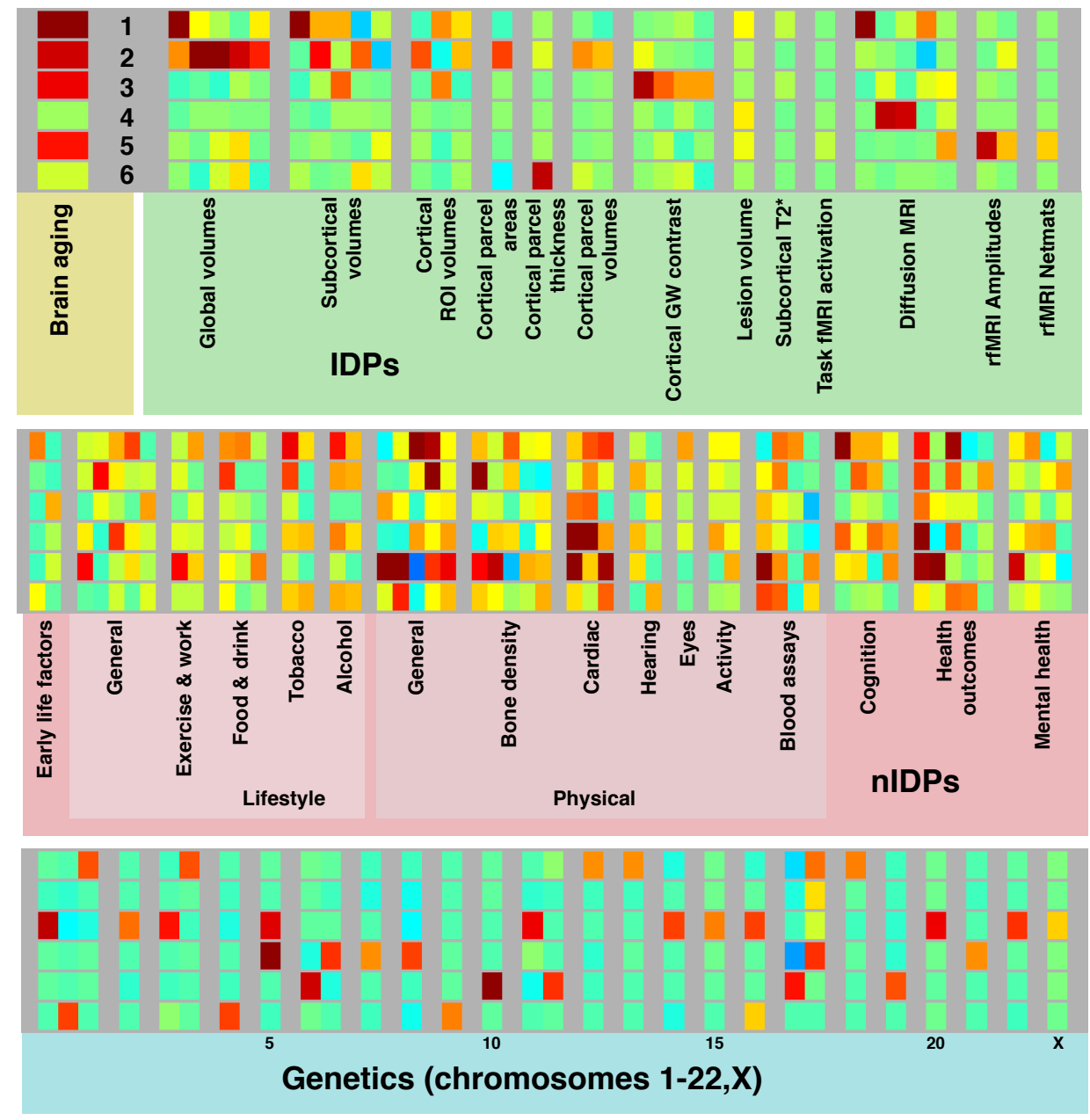

Figure A.14: Mapping of brain-aging mode-clusters onto classes of IDPs, nIDPs and chromosomes. This is an expansion of Fig. 2 (lower), to also show how the 6 brain-aging mode-clusters map onto non-imaging measures and genetics. 
bioRxiv preprint doi: https://doi.org/10.1101/802686; this version posted January 18,2020 . The copyright holder for this preprint (which was not certified by peer review) is the author/funder, who has granted bioRxiv a license to display the preprint in perpetuity. It is made available under aCC-BY 4.0 International license.
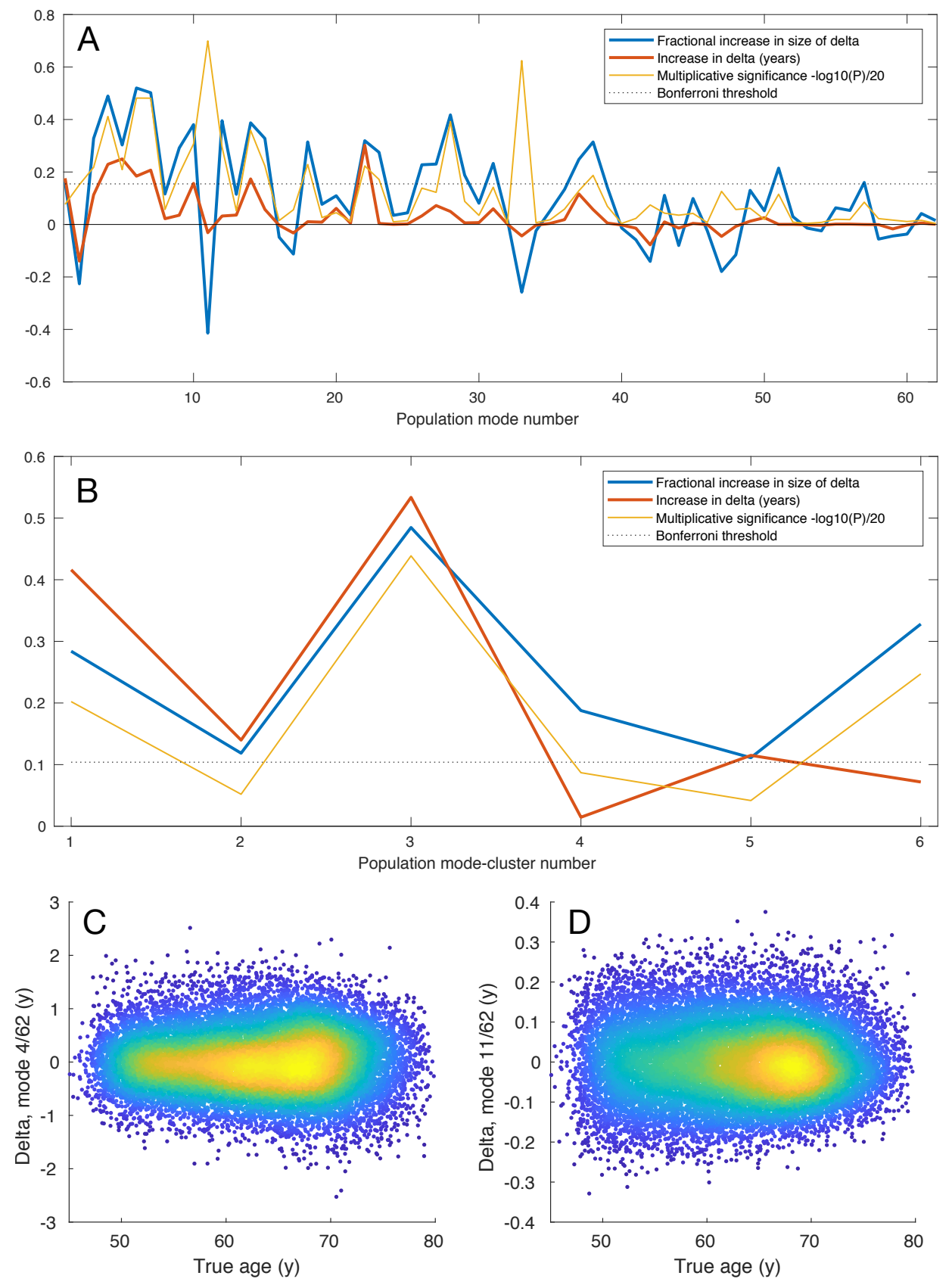

Figure A.15: Non-additive modelling of brain-aging. Non-additive modelling shows where modes and mode-clusters have the scale of brain-age delta changing as a function of age (see Methods). In most cases (e.g., C, mode $4_{62}$ ), delta is either constant or increases with aging. In a few cases (e.g., D, mode $11_{62}$ ), delta is decreasing with age. 